\title{
Channel Coding for Progressive Images in a 2-D Time-Frequency OFDM Block With Channel Estimation Errors
}

\author{
Laura Toni, Student Member, IEEE, Yee Sin Chan, Member, IEEE, Pamela C. Cosman, Fellow, IEEE, and
} Laurence B. Milstein, Fellow, IEEE

\begin{abstract}
Coding and diversity are very effective techniques for improving transmission reliability in a mobile wireless environment. The use of diversity is particularly important for multimedia communications over fading channels. In this work, we study the transmission of progressive image bitstreams using channel coding in a 2-D time-frequency resource block in an OFDM network, employing time and frequency diversities simultaneously. In particular, in the frequency domain, based on the order of diversity and the correlation of individual subcarriers, we construct symmetric $n$-channel FEC-based multiple descriptions using channel erasure codes combined with embedded image coding. In the time domain, a concatenation of RCPC codes and CRC codes is employed to protect individual descriptions. We consider the physical channel conditions arising from various coherence bandwidths and coherence times, leading to a range of orders of diversities available in the time and frequency domains. We investigate the effects of different error patterns on the delivered image quality due to various fade rates. We also study the tradeoffs and compare the relative effectiveness associated with the use of erasure codes in the frequency domain and convolutional codes in the time domain under different physical environments. Both the effects of intercarrier interference and channel estimation errors are included in our study. Specifically, the effects of channel estimation errors, frequency selectivity and the rate of the channel variations are taken into consideration for the construction of the 2-D time-frequency block. We provide results showing the gain that the proposed model achieves compared to a system without temporal coding. In one example, for a system experiencing flat fading, low Doppler, and imperfect CSI, we find that the increase in PSNR compared to a system without time diversity is as much as $9.4 \mathrm{~dB}$.
\end{abstract}

Index Terms-Channel coding, cross-layer design, diversity, multimedia communications, multiple description coding, orthogonal frequency division multiplexing (OFDM), progressive transmission, wireless video.

\section{INTRODUCTION}

$\mathbf{I}$ $\mathrm{N}$ recent years, with the rapid convergence of multimedia, Internet and wireless communications, there is a growing trend of heterogeneity [in terms of channel bandwidths, mobility

Manuscript received July 21, 2008; revised May 25, 2009. First published July 06, 2009; current version published October 16, 2009. This work was supported in part by the Center for Wireless Communications at UCSD, in part by the National Science Foundation under Grant CCF-0635165, and in part by the UC Discovery Grant Program. The associate editor coordinating the review of this manuscript and approving it for publication was Dr. Amy R. Reibman.

L. Toni is with TERA, Italian Institute of Technology (IIT), Genova, Italy, and also with the WiLab, University of Bologna, Bologna, Italy (e-mail: laura. toni@iit.it).

Y. S. Chan is with Corporate Technology, Verizon, Walnut Creek, CA 94597 USA (e-mail: chanys@ieee.org).

P. C. Cosman and L. B. Milstein are with the Department of Electrical and Computer Engineering, University of California at San Diego, La Jolla, CA 92093-0407 USA (e-mail: pcosman@code.ucsd.edu; milstein@ece.ucsd.edu).

Digital Object Identifier 10.1109/TIP.2009.2026676 levels of terminals, end-user quality-of-service (QoS) requirements] for emerging integrated wired/wireless networks. Embedded source coding, allowing partial decoding at various resolution and quality levels from a single compressed bitstream, is a promising technology for multimedia communications. However, embedded source coders are usually extremely sensitive to channel impairments which can be severe in mobile wireless links due to multipath signal propagation, delay and Doppler spreads, and other effects. Sometimes a single error can cause an unrecoverable loss in synchronization between encoder and decoder, and produce substantial quality degradation.

Early studies of embedded transmission include [1] and [2]. Both papers studied the transmission of a progressively compressed bitstream employing the Set Partitioning in Hierarchical Trees (SPIHT) source coder combined with rate-compatible punctured convolutional (RCPC) codes. Coding and diversity are very effective techniques for improving the transmission reliability in a mobile wireless environment. However, time diversity achieved by channel coding plus intrapacket interleaving in a single carrier (SC) communication system becomes less effective in a slow fading environment where correlated and prolonged deep fades often result in the erasure of the whole packet or even several contiguous packets. Hence, although improvement could still be achieved due to the coding gain associated with the use of RCPC codes, the performance was not satisfactory [2].

To improve the performance against deep fades in a wireless environment, two approaches have been proposed to exploit diversity in the time domain at the physical layer for SC communication systems. One was to add systematic Reed-Solomon (RS) codes across multiple packets [3]. Specifically, channel codes consisted of a concatenation of RCPC and CRC codes as the row codes and RS codes as the column codes. With the addition of RS codes across multiple packets, lost packets might still be recoverable due to independently faded time slots [3].

Another approach [4]-[7] uses contiguous information symbols from the progressive bitstreams, which, instead of being packed in the same packets [1], [3], are spread across multiple packets (descriptions). The information symbols are protected against channel errors using systematic RS codes, with the level of protection depending on the relative importance of the information symbols. This coding scheme is sometimes referred to as symmetric $n$-channel FEC-based multiple description (MD) coding. Due to the individually decodable nature of the multiple packets, the source can be recoverable despite packet loss, although at a lower fidelity that depends on the number of successfully received packets. Analogous to the physical layer diversity 
techniques offered by channel coding, this has sometimes been referred to as application layer diversity [8].

While both approaches perform well in slow fading environments, the order of diversity of the physical channel is vital to the selection of system parameters (e.g., choice of channel codes and corresponding channel code rates) as shown in [9]. Despite their importance, such factors are usually overlooked in the literature. More importantly, studies of these channel coding techniques have been limited to 1-D time domain coding in a slow fading environment [3], [7]. For fast fading, rapid channel variations due to high mobility can potentially provide a high diversity gain and significantly improve the effectiveness of channel coding in the time domain. Unfortunately, rapid channel variation also poses a significant challenge for channel estimation [10]-[12]. The accuracy of this channel state information (CSI) is particularly important in optimizing channel coding. In particular, it has been shown that imperfect CSI due to estimation errors degrades the performance of communications systems designed to take advantage of the diversity opportunities [13]-[15].

In recent years, orthogonal frequency division multiplexing (OFDM) has drawn intense interest. OFDM differentiates itself from a SC communications system in many ways, such as robustness against frequency-selective fading. Frequency diversity by adding redundancy in the frequency domain can combat channel errors due to multipath fading and achieve a more reliable overall system performance. In other words, OFDM offers a unique opportunity to improve system efficiency by employing both time and frequency domain channel coding depending on the propagation environment and user's mobility. A highly scattered environment may make the frequency domain coding more effective. A highly mobile user will probably make time domain coding more compelling. Although there have been some works investigating transmission of embedded bitstreams over OFDM networks [16]-[18], none has explicitly characterized the time and frequency domains independently and jointly optimized the coding scheme based on these different physical environments.

In this work, we study the transmission of progressively coded image bitstreams using channel coding in a 2-D time-frequency resource block in an OFDM network under different physical environments. By properly decoupling the time domain and frequency domain channel variations, we propose a 2-D channel coding scheme which employs time and frequency diversities simultaneously. In particular, in the frequency domain, based on the order of diversity, we construct FEC-based multiple descriptions using channel erasure codes combined with embedded source coding. In the time domain, concatenated RCPC codes and CRC codes protect individual descriptions. Both the effects of intercarrier interference (ICI) and channel estimation errors, which may become severe in a fast fading environment, are taken into consideration. We use pilot symbol assisted modulation (PSAM) with pilot symbol density depending on the channel selectivities in both time and frequency. As diversity is the primary factor determining the performance of a wireless system, the results presented can provide some design criteria for other progressive transmission coding schemes over mobile wireless networks.
The remainder of this paper is organized as follows: In Section II, we give a description of the OFDM system and the channel mode. We also describe the proposed transmission system and discuss some of the issues associated with the use of channel coding in a time-frequency block. Both imperfect channel estimation and intercarrier interference due to rapid channel variations are discussed in Section III. In Section IV, we describe the optimization problem. In Section V, we provide simulation results and discussion. Finally, in Section VI, we provide a summary and conclusion.

\section{Channel Model And Time-Frequency ChANNEL CODING}

The basic principle of OFDM is to split a high-rate data stream into a number of lower rate streams that are transmitted over overlapped but orthogonal subcarriers. Since the symbol duration increases for the lower rate parallel subcarriers, the relative amount of dispersion in time caused by multipath delay spread is decreased. Depending on the propagation environment and the channel characteristics, the resource block in an OFDM system can be used to exploit time and/or frequency diversities through channel coding. For time diversity, channel coding plus interleaving can be used in the time domain. However, for the technique to be effective, the time frame has to be greater than the channel coherence time $(\Delta t)_{c}$. The maximum time-diversity gain $\mathcal{D}_{t}$ is given by the ratio between the duration of a time frame and $(\Delta t)_{c}$.

In addition to time diversity, frequency diversity by adding redundancy across the subcarriers can be applied to combat channel errors. Generally, the maximum achievable frequency diversity $\mathcal{D}_{f}$ is given by the ratio between the overall system bandwidth $W_{T}$ and the coherence bandwidth $(\Delta f)_{c}$.

In this work, we consider a frequency-selective environment and use a block fading channel model to simulate the frequency selectivity [19]. With this model, the spectrum is divided into blocks of size $(\Delta f)_{c}$. Subcarriers in different blocks are considered to fade independently; subcarriers in the same block experience identical fades. As illustrated in Fig. 1, we assume an OFDM system with an overall system bandwidth $W_{T}$, such that we can define $N$ independent subbands. Each subband consists of $M$ correlated subcarriers spanning a total bandwidth of $(\Delta f)_{c}$. The total number of subcarriers in the OFDM system is $N M$. In the time domain, we assume the channel experiences Rayleigh fading. We use the modified Jakes' model [20] to simulate different fading rates, resulting in different time diversity orders.

Fig. 2 illustrates the proposed scheme for transmission of an embedded bitstream over a mobile channel characterized by a doubly selective environment. In the frequency domain, $N_{t}=N M$ symmetric descriptions of approximately equal importance are constructed in which contiguous information from the embedded bitstream is spread across the multiple descriptions/packets [4], [5]. The algorithm adopted for the MD encoding is the one presented in [5]. In particular, because of the progressive nature of the encoded bitstream, MD encoding based on the priority of the bits in the bitstream is adopted, and the optimization of the FEC allocation in the frequency 


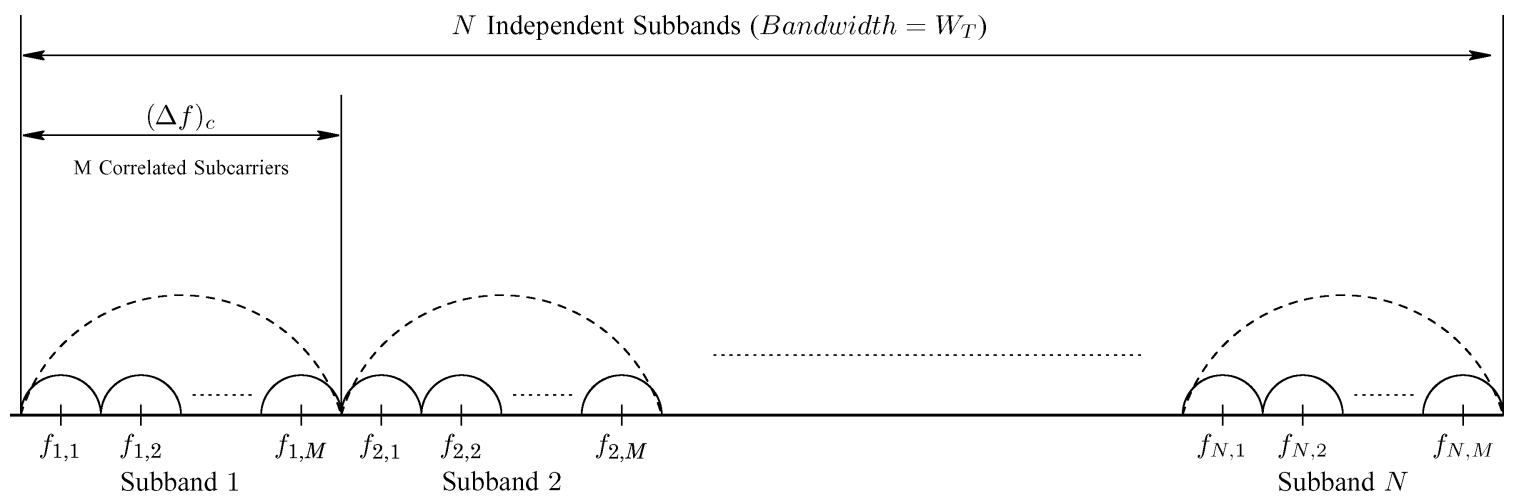

Fig. 1. Subcarrier spectrum assignment.

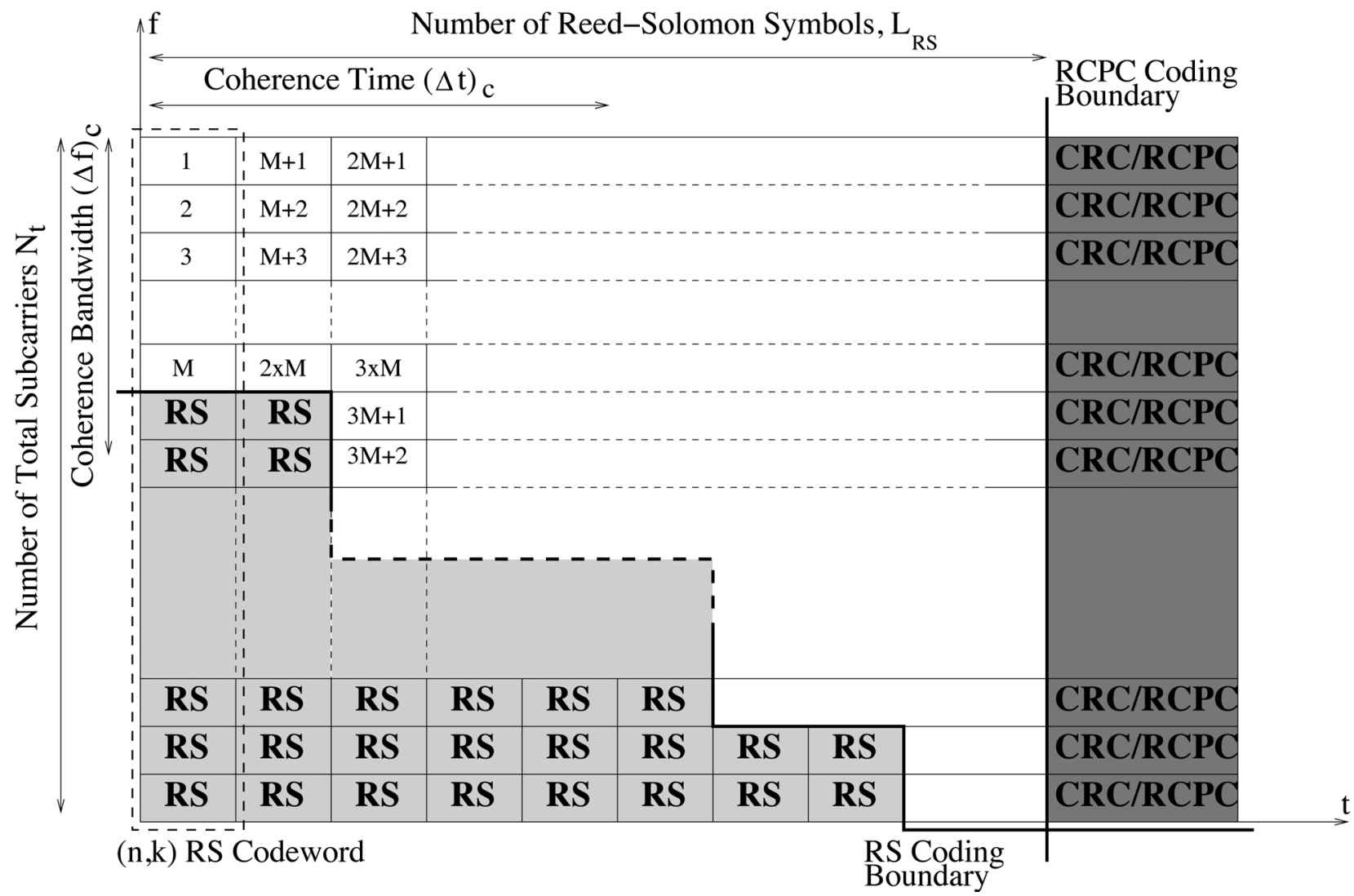

Fig. 2. Transmission of the embedded bitstream over OFDM mobile wireless networks. The dark shaded area to the right of the RCPC coding boundary line represents the bits for CRC and RCPC coding. The lighter shaded area under the RS coding boundary staircase represents Reed-Solomon parity symbols. The unshaded area represents information symbols. Note that the CRC/RCPC parity symbols are interleaved with the RS symbols in the actual system. $N_{t}=N \times M$ total subcarriers and $L_{\mathrm{RS}}$ Reed-Solomon symbols are considered. For each $(n, k)$ RS codeword, $k$ information symbols are encoded into $n=N_{t}$ total symbols.

domain is designed to minimize the expected distortion. Specifically, the information symbols are protected by systematic $(n, k)$ RS codes, ${ }^{1}$ with the level of protection depending on the relative importance of the information symbols, as well as on the order of diversity available in the frequency domain. Generally, an $(n, k)$ MDS erasure code can correct up to $n-k$ erasures. Hence, if any $g$ out of $n$ descriptions are received, those codewords with minimum distance $d_{\min } \geq n-g+1$ can be decoded. As a result, decoding is guaranteed at least up to distortion $D\left(R_{g}\right)$, where $D\left(R_{g}\right)$ refers to the distortion achieved with $R_{g}$ information symbols.

${ }^{1}$ In each $(n, k)$ RS codeword, $k$ information symbols are encoded into $n$ total symbols. While $k$ is variable, $n$ is constant in each codeword and equals $N_{t}$.
The individual descriptions are then mapped to the $N_{t}=N M$ subcarriers. A concatenation of CRC codes and RCPC codes, for possible diversity and coding gains in the time domain, are applied to each description. Since the descriptions are approximately equally important, RCPC codes with the same channel code rate can be applied to protect each individual description. This results in a vertical boundary (RCPC coding line), as illustrated in Fig. 2. The $L_{\mathrm{RS}}$ symbols on the left of the boundary are the RS symbols, while those on the right are CRC/RCPC parity symbols. It should be noted that the multiple description RS symbols and RCPC parity symbols would be interleaved in an actual system. However, for illustration, we show the de-interleaved version throughout 
TABLE I

FACTORS AFFECTING THE APPLICATION LAYER QUALITY-OF-SERVICE (QoS) IN A FAST FADING ENVIRONMENT

\begin{tabular}{l|l} 
Pros & Cons \\
\hline Higher orders of time domain diversity & Larger channel estimation errors \\
$\Rightarrow$ higher coding gain & $\Rightarrow$ Lower channel decoding efficiency \\
$\Rightarrow$ higher diversity gain & Higher level crossing rates \\
& $\Rightarrow$ Errors scattered across multiple packets \\
& $\Rightarrow$ Lower application layer throughput
\end{tabular}

the paper so that the relative amounts of RCPC parity symbols and RS symbols can be clearly indicated.

Since both forms of diversity are not necessarily simultaneously available at any given instant of time, the channel coding scheme should be designed to synergistically exploit the available diversity. For example, in a slow fading environment, channel coding plus interleaving is usually ineffective, especially for delay-sensitive applications such as real-time multimedia services. Hence, in this case, frequency diversity techniques may be more effective than time diversity techniques.

As stated previously, traditional studies of progressive transmission have concentrated on slow fading channels. In fact, in addition to the performance differences in channel coding efficiencies and channel estimation accuracies, the error patterns for different fade rates also affects the application layer throughputs, and, hence, the end-user delivered quality. In particular, in a fast fading environment, the errors are more scattered among multiple packets due to the higher level crossing rate which measures how often the fading crosses some threshold [21]. However, for a slow fading environment, the errors appear more bursty. Consequently, the application layer throughput, measured by the number of successively transmitted packets, in a fast fading environment can be dramatically lower than that in a slow fading system. In Table I, we summarize the factors affecting the selection of an optimal channel coding scheme and end-user performance due to different fading rates.

On the other hand, information on frequency diversity can assist a source-channel codec in selecting a more robust source-cannel coding scheme [9]. For example, while unequal error protection (UEP) is considered as primarily important for robustness for some of the progressive transmission schemes proposed in the literature (e.g., [7]), it was shown that in a highly frequency selective environment, UEP only provides marginal improvement over equal error protection (EEP), while in a frequency diversity deficient system, UEP can greatly improve the performance of progressive transmission over an OFDM system.

\section{ICI AND CHANNEL ESTIMATION ERRORS}

The assumptions of perfect channel estimation and orthogonality between subcarriers cannot be considered accurate for fast fading environments. Rapid channel variations may cause severe ICI [22]-[24] and channel estimation errors, thereby degrading overall system performance. In this work, we model the ICI as in [22], i.e., a zero mean Gaussian random process with variance $\sigma_{I C I}^{2}$ expressed as

$$
\sigma_{I C I}^{2}=E_{s}-\frac{E_{s}}{N_{t}^{2}}\left\{N_{t}+2 \sum_{i=1}^{N_{t}-1}\left(N_{t}-i\right) J_{0}\left(2 \pi f_{n d} i\right)\right\}
$$

where $E_{s}$ is the modulated symbol energy, $N_{t}$ is the number of subcarriers in the OFDM system, $f_{n d}$ is the normalized Doppler spread and $J_{0}(\cdot)$ is the zero-order Bessel function of the first kind. The ICI varies directly with the Doppler frequency.

In addition to ICI, channel variations in the time domain may also increase the difficulty in channel estimation. The accuracy of this channel state information (CSI) is particularly important for coherent demodulation and channel decoding. We adopt pilot symbol assisted modulation (PSAM), commonly used in practical OFDM networks [11], [12], [25]-[29]. We refer the reader to [25] for details of PSAM and the analysis of channel estimation errors.

In this work, as shown in Fig. 3(a), pilot symbols are periodically inserted in the transmitted data symbols with a spacing equal to $L$ modulated symbols. At the receiver, a linear minimum mean square error (MMSE) channel estimator [30] is adopted to estimate the fading coefficient using the following procedures: First, pilot symbols are extracted from the received sequence and the associated channel coefficients are evaluated. Then, the channel coefficient at the $l$ th data time $(l \neq j L)$ is estimated by interpolating the $K$ nearest pilot symbols with a Wiener filter. In particular, we consider $K=2$, i.e., each channel parameter is estimated by interpolating the two closest pilot samples. Let us denote the received pilot symbol of the generic $i$ th $\operatorname{slot}^{2}$ as $\widetilde{h}[i L]$ and assume that the $l$ th data symbol is transmitted in this $i$ th slot. Hence, $\widetilde{h}=[\widetilde{h}[i L], \widetilde{h}[(i+1) L]]^{T}$ is the set of two pilot symbols interpolated to estimate the $l$ th channel coefficient $h[l]$. Defining $\hat{h}[l]$ as the estimator of $h[l]$, the channel estimation errors can be expressed as

$$
\varepsilon[l]=h[l]-\hat{h}[l], \quad l=i L \ldots(i+1) L-1 .
$$

The quality of the estimation is expressed in terms of the mean square error $\sigma_{e}^{2}=E\left[\varepsilon^{2}\right]$, where $E[\cdot]$ denotes the expectation operator. Defining $\boldsymbol{w}[l]=E\left[\widetilde{\boldsymbol{h}} h^{*}[l]\right]$ and $\boldsymbol{R}=E\left[\widetilde{\boldsymbol{h}} \widetilde{\boldsymbol{h}}^{\dagger}\right]$, the variance of the channel estimation errors can be expressed as [12] and [25] ${ }^{3}$

$$
\sigma_{e}^{2}[l]=1-\boldsymbol{w}^{\dagger}[l] \boldsymbol{R}^{-1} \boldsymbol{w}[l]
$$

where superscripts $*,{ }^{T}$ and ${ }^{\dagger}$ stand for conjugate, transpose and transpose conjugate respectively. From (3), it can be seen that the estimation error variance depends on the channel correlation function. Recalling that we use a Jakes' model, the time correlation function is $r[n]=E\left[h[l] h^{*}[l+n]\right] \propto J_{0}\left(2 \pi n f_{n d}\right)$. This means that both the correlation function and the estimation error

\footnotetext{
${ }^{2}$ The transmitted bitstream is divided into slots of length equal to the pilot spacing, i.e., L symbols. The first symbol of each slot is a pilot symbol; the other (L-1) symbols are data, as shown in Fig. 3.

${ }^{3} \mathrm{~A}$ multiplicative coefficient (the total average power of the channel impulse response) has been set equal to 1 and, therefore, is ignored.
} 


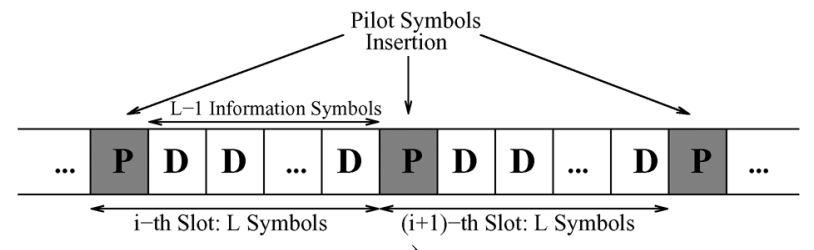

a)

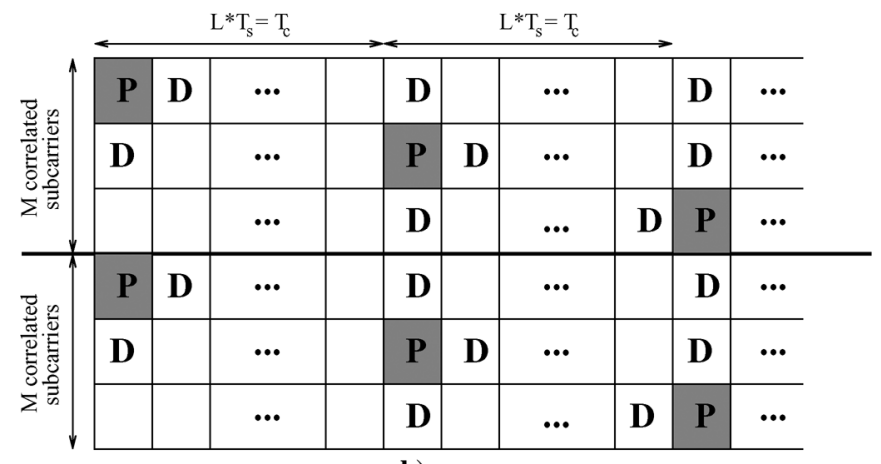

b)

Fig. 3. Pilot insertion scheme for systems with: (a) one correlated subcarrier; (b) $\mathrm{M}$ correlated subcarriers.

variance depend on the normalized Doppler frequency $\left(f_{n d}\right)$. In particular, the channel estimation gets worse when the Doppler frequency increases. It is worthwhile to notice that the variance $\sigma_{e}^{2}[l]$ depends also on the statistics of the received pilot samples $\widetilde{\boldsymbol{h}}$, and thus on the signal-to-noise-ratio (SNR).

In this work, the pilot and data symbols are transmitted at the same power level. Since a frequent pilot insertion improves channel estimation, at the cost of reduced throughput, a fixed pilot scheme for different Doppler frequency environments is not the best solution. Thus, we consider a pilot spacing equal to the coherence time. It follows that for a slow fading channel, the number of pilot symbols is negligible, leading to a high transmitted throughput. In high Doppler systems, to achieve good channel estimation, we have to reduce significantly the number of transmitted data symbols in each packet. Since under the block fading model, correlated subcarriers experience the same fading channel in the frequency domain, pilot symbols inserted once every coherence time are distributed among correlated subcarriers, as shown in Fig. 3(b). Thanks to this pilot scheme, the number of inserted pilot symbols decreases drastically in systems with low frequency diversity order.

\section{PROBLEM FORMULATION}

In this section, we describe the optimization problem to be solved. Consider $N$ i.i.d. subbands, each with $M$ subcarriers and packet size equal to $L_{\mathrm{RS}}$ code symbols before channel coding using RCPC/CRC codes. Since each vertical column corresponds to one RS codeword, there are altogether $L_{\mathrm{RS}} \mathrm{RS}$ codewords. The constraint on the bit budget/packet can then be written as

$$
N_{t} \times\left(L_{\mathrm{RS}} \times B_{\mathrm{RS}}+B_{\mathrm{CRC}}\right) / R_{\mathrm{rcpc}} \leq B_{\mathrm{tot}}
$$

where $B_{\mathrm{CRC}}$ is the bit budget allocated in each description for the CRC codes and $R_{\text {rcpc }}$ is the channel code rate of the RCPC codes. $B_{\mathrm{RS}}$ is the number of bits-per-RS symbol and $B_{\text {tot }}$ is the total bit budget of the RB.

We assume that for RS codeword $l$, where $l \in\left[1, L_{\mathrm{RS}}\right], c_{l}$ code symbols are assigned to information data symbols. Hence, the number of RS parity symbols assigned to codeword $l$ is

$$
f_{l}=N_{t}-c_{l} \quad l \in\left[1, L_{\mathrm{RS}}\right] .
$$

Let $\phi_{t h}$ be the minimum number of descriptions that a decoder needs to reconstruct the source, and $g$ be the number of correctly received packets. The reception of any number of packets $g \geq \phi_{t h}$ leads to improving image quality $D\left(R_{g}\right)$, where $R_{g}$ is the allocated bit budget for the information symbols

$$
R_{g}=\sum_{\left\{l: c_{l} \leq g\right\}} c_{l} \times B_{\mathrm{RS}} .
$$

Hence, the overall RS channel code rate equals $R_{\mathrm{rs}}=$ $R_{N_{t}} /\left(N_{t} \times L_{\mathrm{RS}} \times B_{\mathrm{RS}}\right)$. Given the source code rate-distortion curve $D\left(R_{g}\right)$ and the packet loss probability mass function $P_{\mathcal{J}}(j)$, where $j=N_{t}-g$ is the number of lost packets, we can minimize the expected distortion as follows:

$$
\left.\begin{array}{rl}
E^{*}[D]=\min _{\left\{c_{l}, R_{\mathrm{rcpc}}\right\}}\left\{\sum_{j=0}^{N_{t}-\phi_{t h}} P_{\mathcal{J}}(j) D\left(R_{S_{\mathrm{tot}}-j}\right)\right. \\
+\sum_{j=N_{t}-\phi_{t h}+1}^{N_{t}} P_{\mathcal{J}}(j) D_{0}
\end{array}\right\}
$$

subject to the constraint on the overall bit budget

$$
\frac{R_{N_{t}} / R_{\mathrm{rs}}+\left(B_{\mathrm{CRC}} \times N_{t}\right)}{R_{\mathrm{rcpc}}} \leq B_{\mathrm{tot}}
$$

where $D_{0}$ corresponds to the distortion when fewer than $\phi_{t h}$ descriptions are received, and so the decoder must reconstruct the source without being able to use any of the transmitted information. For a still image, this typically means reconstructing the entire image at the mean pixel value.

The packet loss probability mass function $P_{\mathcal{J}}(j)$ depends on $(\Delta f)_{c},(\Delta t)_{c}$ and $R_{\text {rcpc }}$. Although $P_{\mathcal{J}}(j)$ can be found analytically for uncorrelated fading channels, due to the correlated fading in both time and frequency domains of the mobile environment considered here, we use simulations to find $P_{\mathcal{J}}(j)$. We use the iterative procedure described in [5] to solve the optimization problem (7).

\section{RESUlTS AND DISCUSSION}

We carried out simulations on the $512 \times 512$ gray-scale images Lena, Peppers, and Goldhill. Similar results were obtained for all three. Hence, in this paper, we only present the results using the Lena image. The image was encoded using the SPIHT [31] algorithm to produce an embedded bitstream. The serial bitstream was converted into 128 parallel bitstreams using the FEC-based multiple description encoder. The 128 descriptions were mapped to the OFDM system with 128 subcarriers. We used RS codes in the frequency domain and there were 8 bits per RS symbol. The packet size was set equal to 512 bits, and two bytes per description were appended for the CRC code. We 


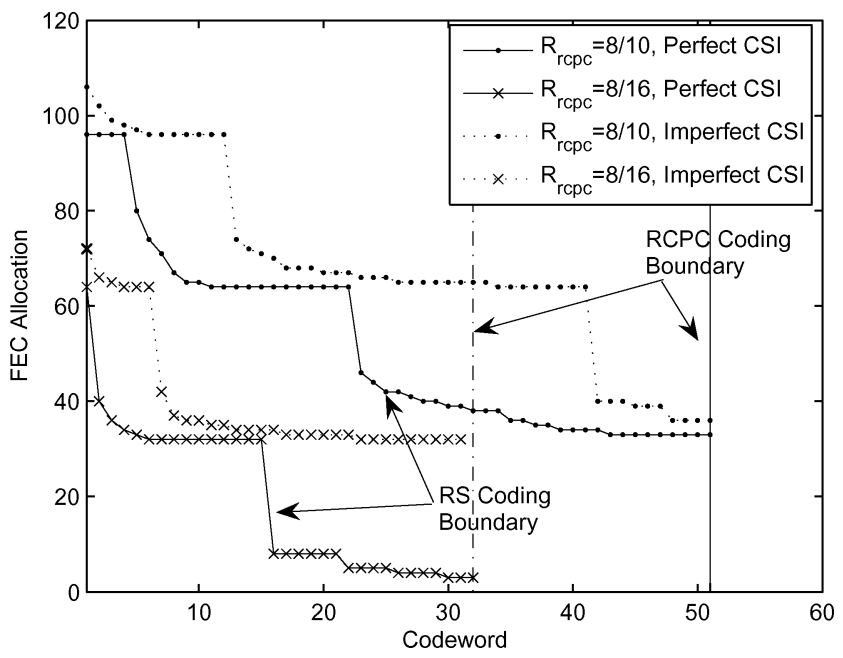

Fig. 4. Profiles showing the optimal allocation of source and channel symbols for systems with $(N, M)=(4,32), f_{n d}=10^{-3}$ and SNR $=16.0 \mathrm{~dB}$ for different choices of RCPC coding rates and for both perfect CSI and imperfect CSI.

used QPSK modulation and considered both perfect and imperfect CSI. It should be noted that more sophisticated modulation schemes could be used to further improve the system performance, such as the one discussed in [32]. However, for the sake of simplicity and better understanding of the fundamental impact of the time and frequency diversities on the construction of the 2-D OFDM block, a fixed modulation scheme using QPSK was chosen. The RCPC codes of rates $R_{\text {rcpc }}=$ $8 / 9,8 / 10, \ldots, 8 / 24$, were obtained by puncturing an $R_{c}=1 / 3$ mother code with $K=7, p=8$ and generator polynomials $(133,165,171)_{\text {octal }}$ with the puncturing table given in [33].

In the following figures, we illustrate the proposed channel coding scheme under different fading environments, and study the effects of channel estimation on the selection by comparing performance of systems with perfect CSI to systems with imperfect CSI and ICI. From here onwards, for systems with imperfect CSI and ICI, ICI is omitted from the notation for the sake of brevity, although it is considered as well. We begin by studying the optimized construction of RS information and parity symbols for:

- different values of RCPC coding rate;

- both perfect and imperfect CSI;

- different frequency diversity orders and different fading rates.

Then we study how the received image PSNR varies for different Doppler spreads, for perfect and imperfect CSI, and for different frequency diversity orders.

In Fig. 4, we show the optimized construction of RS information symbols, RS parity symbols and RCPC parity symbols for different $R_{\text {rcpc }}$ 's for $(N, M)=(4,32)$, normalized Doppler spread $f_{n d}=10^{-3}, \mathrm{SNR}=16 \mathrm{~dB}$, and both perfect CSI and imperfect CSI. The maximum order of diversity achieved in the frequency domain is $\mathcal{D}_{f}=4$, while the maximum order of diversity in the time domain is $\mathcal{D}_{t}=1$. In other words, no diversity can be exploited by using RCPC codes, although coding gain can still be obtained. In general, lower code rates in the time domain improve the packet loss performance, thus

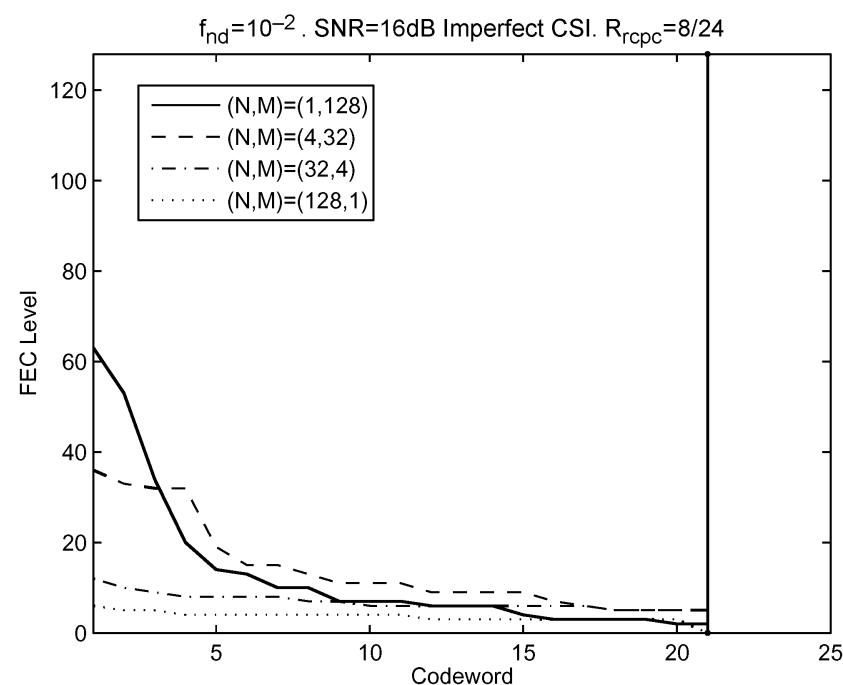

Fig. 5. Profiles showing the optimal allocation of source and channel symbols for systems with $R_{\mathrm{rcpc}}=8 / 24$ and SNR $=16.0 \mathrm{~dB}$ and imperfect CSI for systems with frequency diversity orders $N=1,4,32,128$, respectively.

reducing the number of RS parity symbols required for minimizing the expected distortion $E[D]$, as can be noticed from the figures. Moreover, since for a fixed code rate in the time domain the perfect-CSI system outperforms the imperfect-CSI system, the latter system requires more protection in the frequency domain than does the perfect system.

As can be seen from Fig. 4, the RS code boundaries exhibit similar degrees of tilting for both $R_{\text {rcpc }}$ rates, and for both the perfect CSI and imperfect CSI systems for the same diversity order. As discussed in [9], the degree of tilt of the RS boundary indicates the importance of unequal error protection (UEP) relative to equal error protection (EEP), which has a horizontal RS boundary line. Hence, the results demonstrate that although the packet loss performance of an individual subcarrier can be improved by using a lower channel coding rate, the degree of UEP, represented by the tilt of the RS boundaries, mainly depends on the frequency diversity order of the system, and is relatively insensitive to the selection of the channel code rate in the time domain. In addition to the similar degree of tilting, the curves also show similar stepwise behavior. In particular, the RS boundaries show similar leveling behavior at approximately the same FEC value with step-size roughly equal to the coherence bandwidth. This observation agrees with the simulation results shown in [9], which is mainly due to, in addition to the same diversity order, the perfectly correlated fading within a subband in the frequency domain, which results in, with high probability, the simultaneous loss of the correlated subcarriers when a subband is under a deep fade.

In general, as the frequency diversity order increases, the variation of the number of lost packets decreases and thus reduces the need, and, hence, the relative advantages, of UEP, as shown in Fig. 5. In particular, in Fig. 5, we show the optimal allocation of source and channel symbols for imperfect CSI systems with different frequency diversity orders $(N=1,4,32,128)$ in an environment with $f_{n d}=10^{-2}$. The time domain channel code rate $R_{\text {rcpc }}$ is fixed at $8 / 24$. As can be seen, in spite of significant difference in the time domain channel conditions due to the 


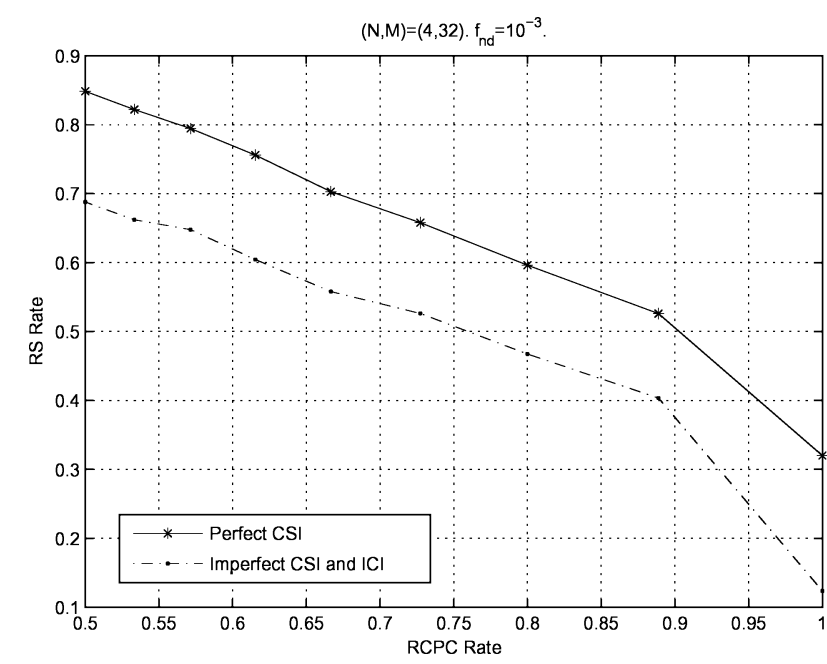

Fig. 6. Optimal $R_{\text {rs }}$ versus $R_{\text {rcpc }}$ for systems with $(N, M)=(4,32), f_{n d}=$ $10^{-3} \mathrm{SNR}=16.0$ and both perfect and imperfect CSI.

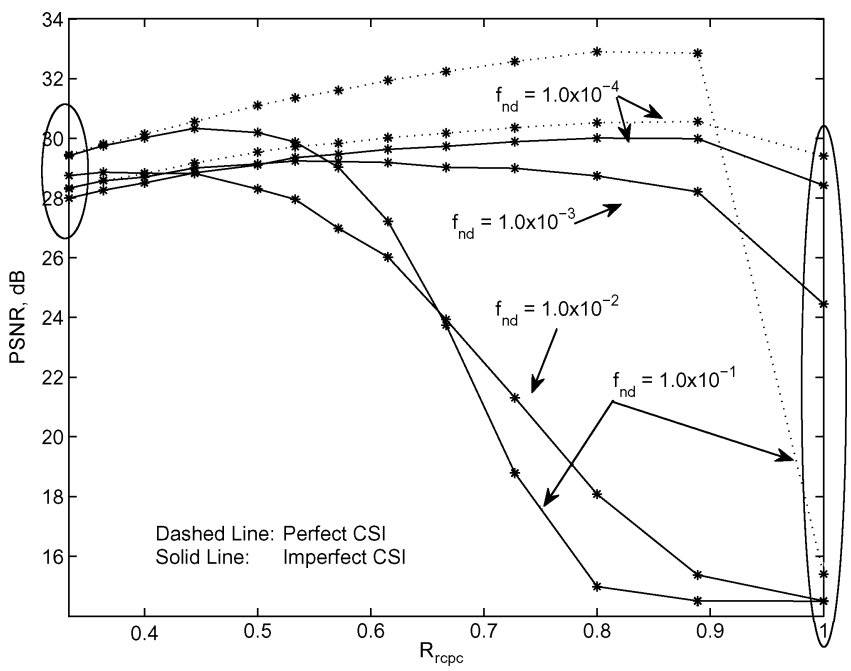

Fig. 7. Optimized PSNR versus $R_{\text {rcpc }}$ for different Doppler spreads in systems with $(N, M)=(4,32), \mathrm{SNR}=16 \mathrm{~dB}$ and imperfect CSI.

effect of fast fading and the time domain channel coding, similar behavior of the RS boundaries can still be observed when compared with the slow fading system reported in Fig. 12 of [9]. Specifically, the amount RS code rate increases with the increasing frequency diversity order, while the degree of UEP decreases with increasing frequency diversity order. Observe that at $N=128$, the RS boundary is almost flat.

The tradeoff between RCPC codes and RS codes for both perfect and imperfect CSI is further illustrated in Fig. 6, where the optimal $R_{\mathrm{rs}}$ versus $R_{\mathrm{rcpc}}$ is shown. By lowering the RCPC code rates, better packet loss performance is achieved due to the coding gain, and less protection in the frequency domain is required.

In Fig. 7, we plot the optimal peak-signal-to-noise ratio (PSNR) performances against $R_{\text {rcpc }}$ for selected normalized Doppler spreads in systems with $(N, M)=(4,32)$, $\mathrm{SNR}=16 \mathrm{~dB}$ and imperfect CSI at the receiver. In the figure, for each selection of the time domain channel coding rate $R_{\text {rcpc }}$, the RS boundary is optimally constructed to maximize the delivered PSNR based on the frequency diversity order of the system. For comparison, in the plot, we also include the curves for the normalized Doppler spread with $f_{n d}=10^{-1}$ and $10^{-4}$ with perfect CSI, representing the fast fading and slow fading scenarios with ideal channel estimation. As can be observed, the curve corresponding to $f_{n d}=10^{-4}$ with perfect CSI tracks the performance of the system with imperfect CSI closely, with minor degradation due to channel estimation errors. However, the curve corresponding to $f_{n d}=10^{-1}$ and imperfect CSI deviates significantly from the system with perfect CSI due to the high channel estimation errors in a fast fading environment. It is worth mentioning that for the fast fading $f_{n d}=10^{-1}$ environment and high signal-to-noise ratio, the system with imperfect CSI performs close to the perfect CSI system, indicating that extra redundancy can effectively compensate for channel estimation errors in a highly mobile scenario. Note that if the signal-to-noise ratio is low, even the lowest channel code rate in the time domain cannot sufficiently compensate for the effects of channel estimation errors.

Perhaps the more interesting observation is the crossovers among the curves with different fade rates. To explain crossovers, we look at the two extremes of the plot, i.e., the highest and lowest time domain channel code rate $R_{\text {rcpc }}=1$ and $R_{\text {rсpc }}=0.333$. For the selected fade rates, at $R_{\text {rсpc }}=0.333$, the PSNR performance increases monotonically with the fade rate, while the PSNR performance at $R_{\text {rcpc }}=1$ shows a monotonic decreasing behavior with increasing fade rate. The different behaviors are due to the two countering effects on the system performance as a result of increasing fade rate. As stated previously, on the one hand, the increase in fade rate increases the diversity order in the time domain and, hence, the efficiency of the RCPC channel coding. However, on the other hand, due to the higher level crossing rate in a fast fading system, errors are scattered across multiple packets rather than being bursty. For systems with little or no channel coding in the time domain, this scattered nature of the error pattern can significantly increase the packet loss rate and reduce the application layer throughput. Consequently, the PSNR performance drops drastically, as can be noticed by the significant performance degradation for $f_{n d}=10^{-1}$ and $10^{-2}$. As we shall see below, due to the higher level crossing rate associated with fast fading environments, the correct selection of an RCPC rate is more important for a fast fading system than for a slow fading system.

To further illustrate the effect of the error pattern on the PSNR performance, in Fig. 8, we plot the optimized PSNR performance versus the normalized fading speed $\left(f_{n d}\right)$ for $R_{\text {rcpc }}=1$ for a system with a frequency diversity order $N=16$ and $\mathrm{SNR}=16 \mathrm{~dB}$. We include the PSNR performance curves for both perfect CSI and imperfect CSI. As expected, the performance difference between perfect CSI and imperfect CSI widens as the fade rate increases due to the increasing channel estimation errors. However, more importantly, both curves show a monotonic decreasing behavior with an increasing fade rate due to the increasingly scattered error pattern. In the Appendix, we provide some further analysis for the packet error rates due to the effects of error patterns resulting from different fading rates on the application layer throughput. In particular, by combining 


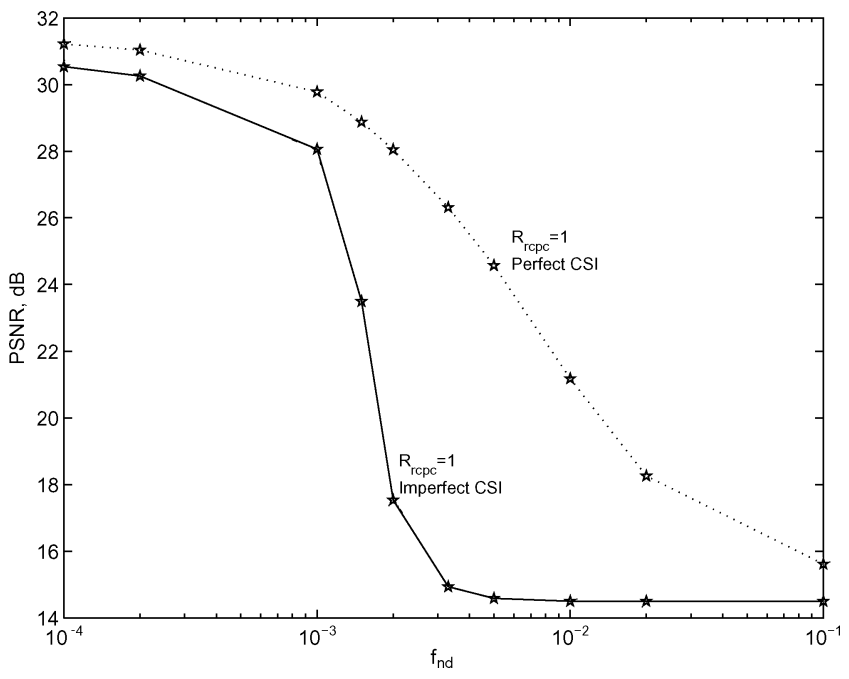

Fig. 8. Optimized PSNR versus $f_{n d}$ for systems with $(N, M)=(16,8)$, $\mathrm{SNR}=16 \mathrm{~dB}$ and $R_{\mathrm{rcpc}}=1$ for perfect and imperfect CSI systems.

the threshold model [34] and the analysis on fade duration distribution [35], [36], we provide a simple analytic solution showing that for an uncoded system, the application layer throughput decreases exponentially with increasing fade rates due to the fact that deep fading events are shorter but occur more frequently.

In Fig. 9(a) and (b), we illustrate the effects of the frequency and time diversity orders on the selection of optimal coding schemes. In Fig. 9(a), we show the optimal PSNR performance versus $R_{\text {rcpc }}$ for different diversity orders $(N=1,2, \ldots, 128)$ in a system with $f_{n d}=10^{-3}$, SNR $=16 \mathrm{~dB}$ and imperfect CSI. In the figure, we also mark with a circle (o) the optimal $R_{\text {rcpc }}$. As the system experiences low Doppler spread with $\mathcal{D}_{t}=1$ and channel estimation becomes more accurate, the selection of optimal coding schemes is dominated by the frequency diversity order of the system. As can be observed, generally a better performance can be achieved with a higher diversity order. More importantly, as the diversity order $N$ increases, the optimal $R_{\text {rcpc }}$ increases and the delivered image quality improves accordingly. Notice also that, except for the case $N=1$, the PSNR performance curves are relatively flat around the optimal $R_{\text {rсpc }}$. To give a specific example, consider the PSNR performance curve for $N=8$. Although $R_{\text {rсpc }}=0.62$ gives the optimal performance, if $R_{\text {rcpc }}=0.5$ or 0.87 is selected instead, only minor degradation is suffered. This is because, in a slow fading environment, the performance loss due to the nonoptimal selection of $R_{\text {rcpc }}$ is partly compensated by the RS coding in the frequency domain. The results indicate that in a slow fading environment, by using the proposed 2-D coding scheme, the results are relatively insensitive to the selection of $R_{\mathrm{rcpc}}$, which can be selected over a broad range. The sub-optimal approach only sacrifices marginal performance degradation. The case $N=1$ represents a flat fading environment, in which RS coding across the subcarriers becomes ineffective.

In Fig. 9(b), instead of a slow fading environment, we study the performance of systems under fast fading conditions. Specifically, we plot the optimal PSNR performance versus $R_{\mathrm{rcpc}}$ for different frequency diversity orders for a fast fading system with $f_{n d}=10^{-1}, \mathrm{SNR}=16 \mathrm{~dB}$ and imperfect CSI. As can be

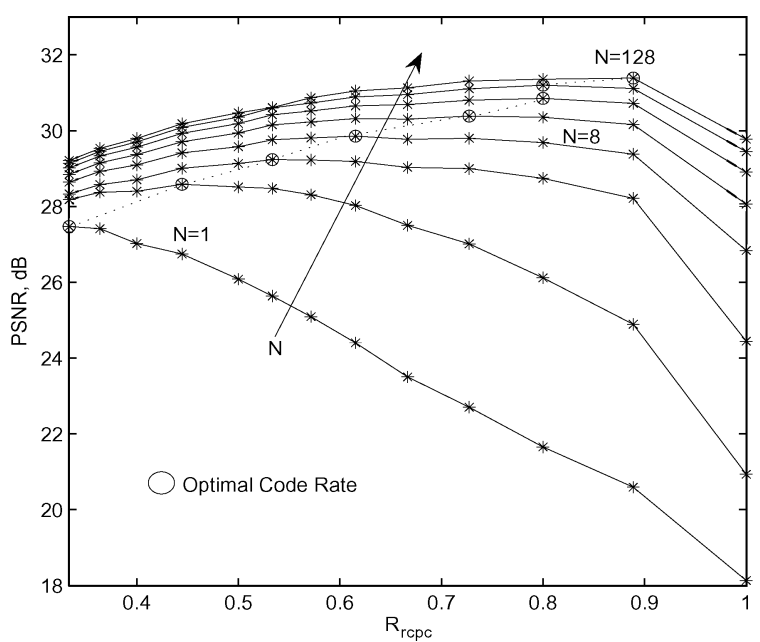

(a)

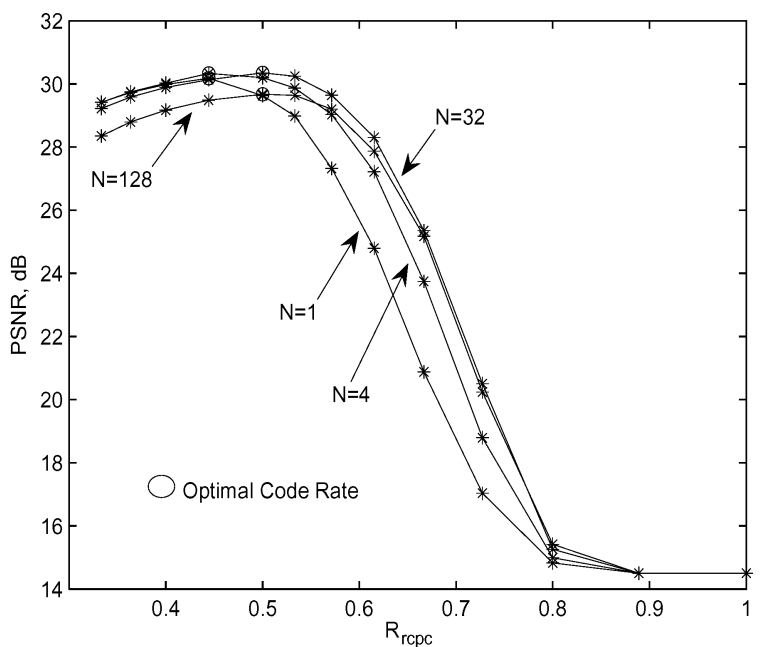

(b)

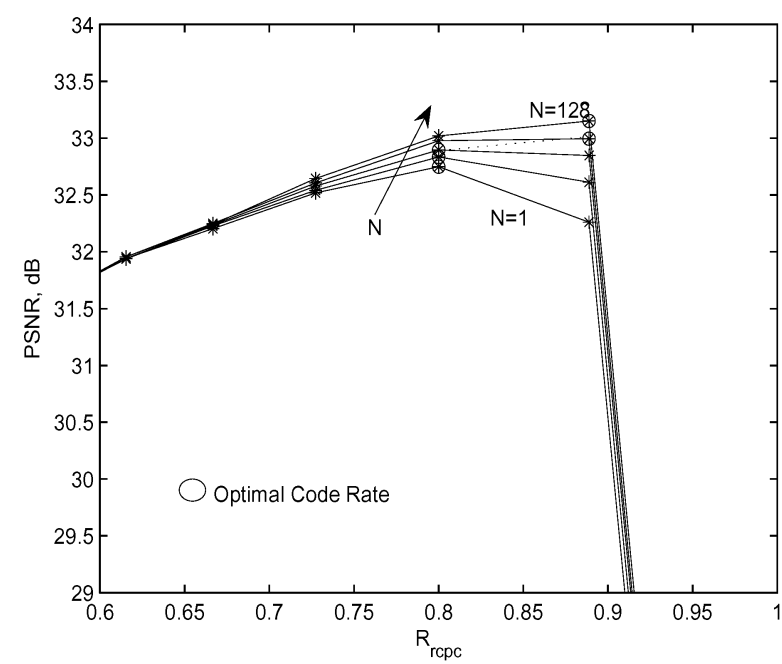

(c)

Fig. 9. Optimized PSNR versus $R_{\text {rcpc }}$ for different coherence bandwidths. (a) $f_{n d}=10^{-3}, \mathrm{SNR}=16 \mathrm{~dB}$ and imperfect CSI. (b) $f_{n d}=10^{-1}$, $\mathrm{SNR}=16 \mathrm{~dB}$ and imperfect CSI. (c) $f_{n d}=10^{-1}$, SNR $=16 \mathrm{~dB}$ and perfect CSI.

easily noticed, by comparing Fig. 9(b) with Fig. 9(a), the performance of a fast fading system is drastically different from 


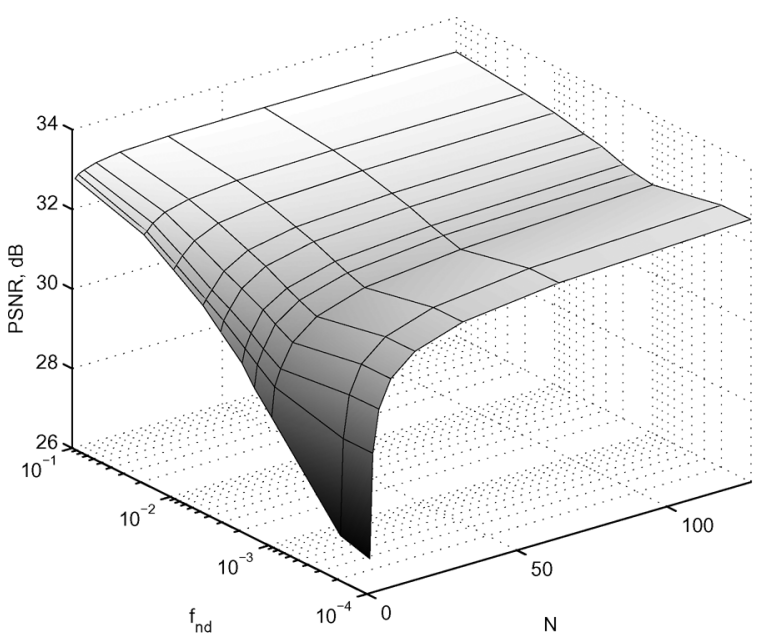

(a)

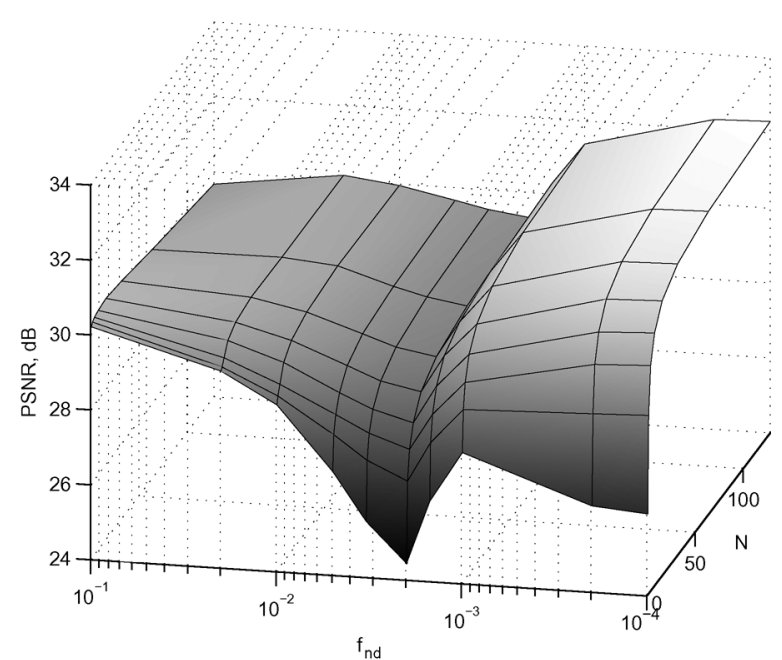

(b)

Fig. 10. Optimal PSNR performances versus both $N$ and $f_{n d}$ in systems with SNR $=16 \mathrm{~dB}$ for both perfect CSI systems (a) and imperfect CSI systems (b).

that of a slow fading system due to a combination of higher diversity, more scattered errors and poorer channel estimation accuracy associated with fast fading environments. In particular, the system experiences a relatively flat region at low $R_{\text {rcpc }}$ rates and a drastic drop in PSNR as it moves towards high $R_{\text {rcpc }}$ Observe that, although the system with a higher frequency diversity order generally provides a better performance, unlike the slow fading systems, the optimal $R_{\text {rcpc }}$ 's are relatively insensitive to the frequency diversity order. This is because in the time domain, the performance is dominated by the high time diversity gain, and thus PSNR depends only slightly on the frequency diversity order. Moreover, it is worth noting that, due to the high time diversity order, the time domain channel coding is very effective and the optimal channel code rate in the time domain is dominated by the channel estimation errors and ICI.

This can be illustrated by comparing Fig. 9(b) with Fig. 9(c). In Fig. 9(c), we plot the corresponding system with perfect CSI as opposed to the system with imperfect CSI shown in Fig. 9(b). Observe that, generally, high $R_{\text {rcpc }}$ 's are preferred for better system performance. However, the performance is relatively insensitive to the frequency diversity order. Moreover, both systems exhibit precipitous drops in PSNR performance due to a more dispersed error pattern, leading to poor application layer throughput if the system is under-protected.

In Fig. 10(a) and (b), we show the optimized PSNR versus both normalized Doppler spread $f_{n d}$ and the number of independent subbands $(N)$ using the proposed coding scheme for a 2-D time-frequency OFDM resource block with perfect and imperfect CSI, respectively. The SNR is set to $16 \mathrm{~dB}$. As can be observed from Fig. 10(a), without channel estimation errors, systems with greater diversity opportunities in the time and/or the frequency domains generally give better performance. However, more importantly, observe the relatively stable performance under different physical environments. Only for both low Doppler and flat fading environments does the system perform poorly. For other values of frequency and time diversity, the PSNR provided is always more than $30 \mathrm{~dB}$, even in the case of systems with low time or low frequency diversity order.

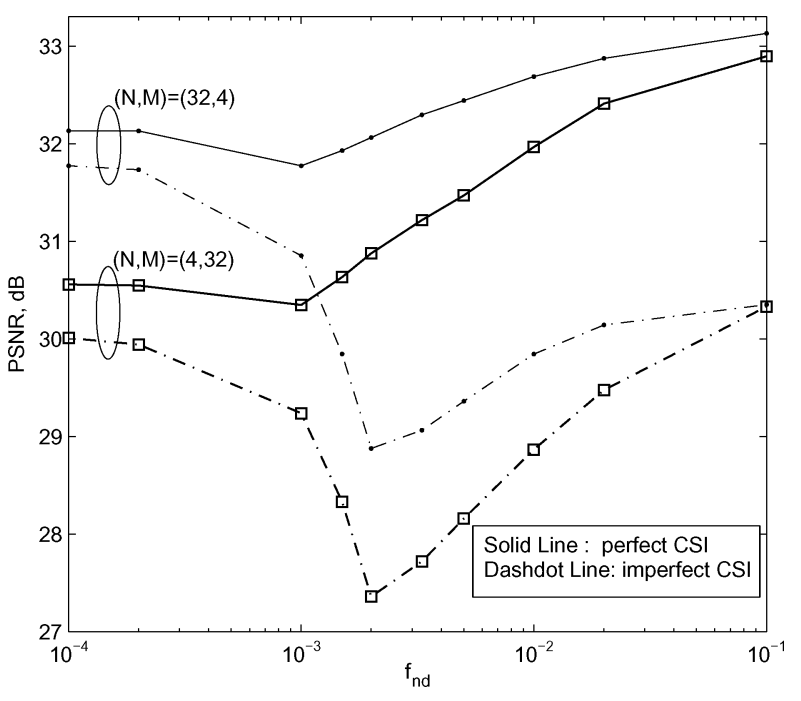

Fig. 11. Optimized PSNR versus $f_{n d}$ for systems with SNR $=16 \mathrm{~dB}$ and both perfect and imperfect CSI. Two different frequency diversity order are considered: $(N, M)=(32,4)$ and $(N, M)=(4,32)$.

In Fig. 10(b), we plot the corresponding system with imperfect CSI. Complicated by the effects of channel estimation errors, the optimal performance becomes more irregular. While, in general, systems with higher frequency diversity orders outperform systems with lower frequency diversity orders, some irregularities are observed in the time domain. In particular, the PSNR drops with decreasing fade rate and starts to rise again at fade rates around $10^{-2}$ and $10^{-3}$. To have a better understanding of the optimal behavior, in Fig. 11, we show the optimal PSNR performance versus $f_{n d}$ for systems with two different frequency diversity orders, $\mathcal{D}_{f}=4$ and $\mathcal{D}_{f}=32$. The SNR is set to $16 \mathrm{~dB}$. The OFDM resource block is constructed with optimal RS profiles and RCPC rates based on the proposed scheme. For comparison, systems with both perfect and imperfect CSI are considered. By first looking at the slow fading section, i.e., the region with $f_{n d}<10^{-3}$, it can be observed that the optimal PSNR performances are relatively flat, with some 


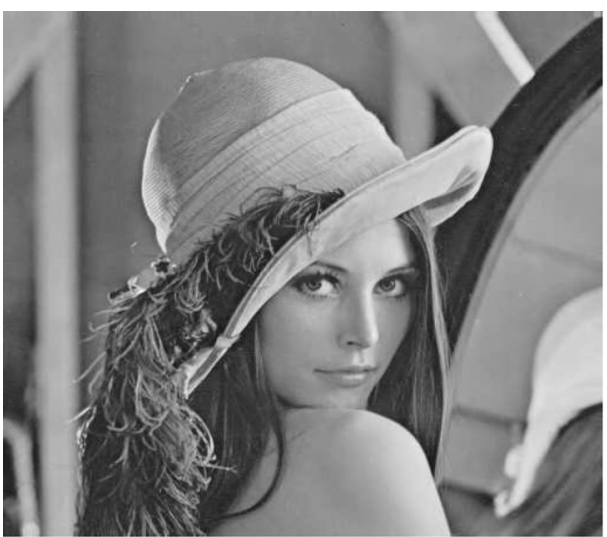

(a)

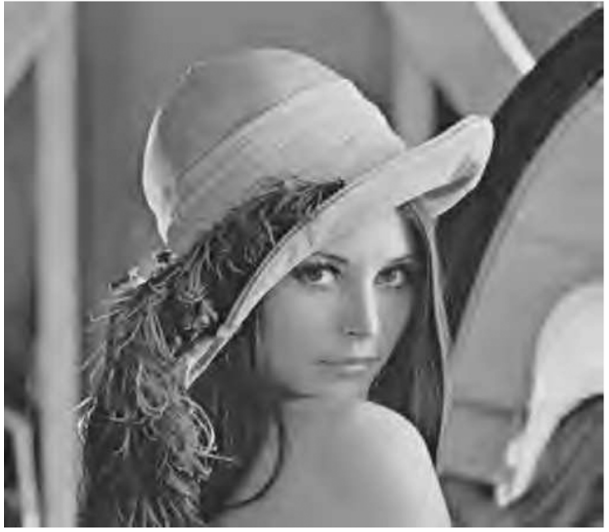

(c)

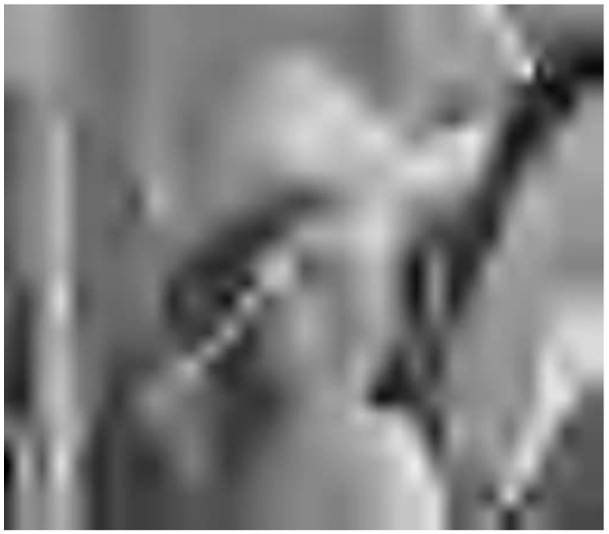

(e)

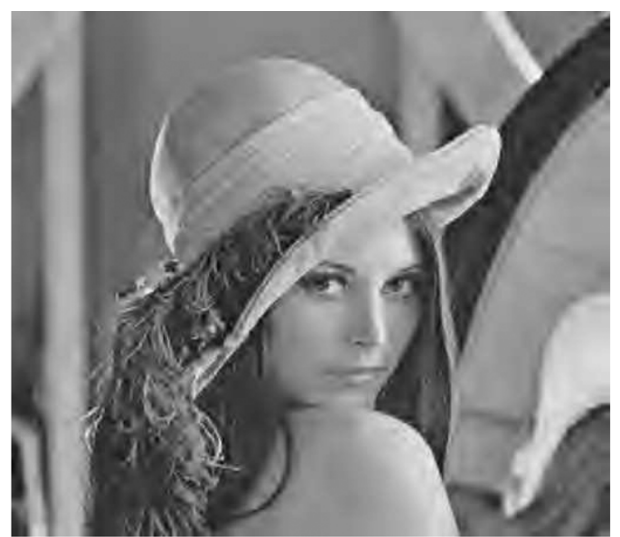

(b)

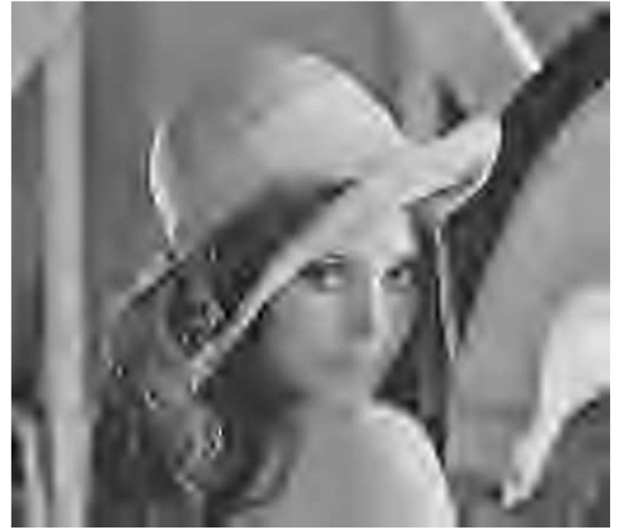

(d)

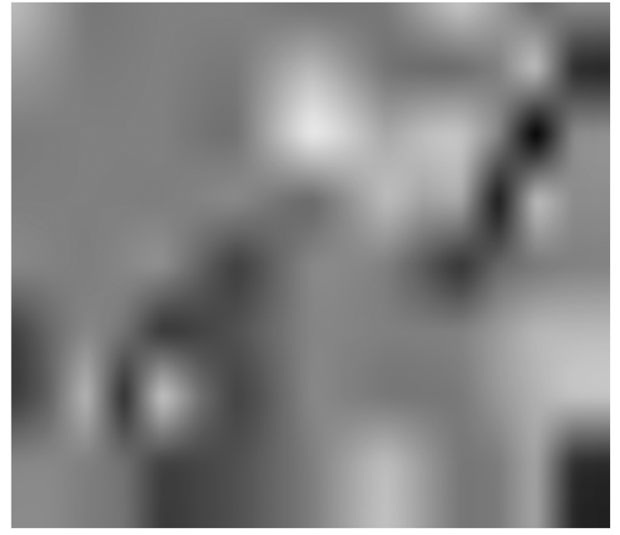

(f)

Fig. 12. Image quality for a system with $f_{n d}=10^{-1},(N, M)=(32,4)$, SNR $=16 \mathrm{~dB}$, and imperfect CSI. We denote by $R_{\mathrm{TX}}$ the transmitted source rate and by $R_{\mathrm{RX}}$ the received source rate. (a) Original image. (b) Received image. $R_{\mathrm{rcpc}}=0.33, R_{\mathrm{rs}}=0.99, \mathrm{PSNR}=29.0 \mathrm{~dB}, R_{\mathrm{TX}}=0.08 \mathrm{bpp}, R_{\mathrm{RX}}=0.073 \mathrm{bpp}$. (c) Received image. Optimal Rate. $R_{\mathrm{rcpc}}=0.5, R_{\mathrm{rs}}=0.89, \mathrm{PSNR}=30.5 \mathrm{~dB}, R_{\mathrm{Tx}}=0.15 \mathrm{bpp}, R_{\mathrm{Rx}}=0.1 \mathrm{bpp}$. (d) Received image. $R_{\mathrm{rcpc}}=0.66$, $R_{\mathrm{rs}}=0.18, \mathrm{PSNR}=25.35 \mathrm{~dB}, R_{\mathrm{TX}}=0.03 \mathrm{bpp}, R_{\mathrm{RX}}=0.02 \mathrm{bpp}$. (e) Received image. $R_{\mathrm{rcpc}}=0.73, R_{\mathrm{rs}}=0.03, \mathrm{PSNR}=20.5 \mathrm{~dB}, R_{\mathrm{TX}}=0.007 \mathrm{bpp}$, $R_{\mathrm{RX}}=0.004$ bpp. (f) Received image. $R_{\mathrm{rcpc}}=1, R_{\mathrm{rs}}=0.008, \mathrm{PSNR}=14.5 \mathrm{~dB}, R_{\mathrm{TX}}=0.001 \mathrm{bpp}, R_{\mathrm{RX}}=10^{-5} \mathrm{bpp}$.

degradation in the systems with imperfect CSI due to channel estimation errors. The performance gap between the perfect CSI and imperfect CSI in this region is relatively small due to better channel estimation accuracy in a slow fading environment. In the middle section, i.e., $10^{-3}<f_{n d}<10^{-2}$, the drops in PSNR performance are steepened for systems with imperfect CSI, due to the combined effects of low time diversity gain, increasing channel estimations errors, and the impact of the more scattered nature of the error pattern. For $f_{n d}>10^{-2}$, the large channel variations provide significant time diversity gain, which improves the efficiency of the RCPC codes and partly compensates for the performance loss due to the channel estimation errors.

Finally, results in terms of reconstructed images in Fig. 12 for systems with $f_{n d}=10^{-1},(N, M)=(32,4)$, and imperfect CSI, provide some subjective comparisons. Here, the advantage of the proposed scheme and the effect of channel impairments under a fast fading environment can be observed by comparing the reconstructed images with the original one. The five reconstructed images [Fig. 12(b)-(f)] represent five dif- 
TABLE II

Optimal PSNR, $R_{\mathrm{rcpc}}, R_{\mathrm{rs}}$, AND $R_{\text {tot }}$ FOR A SYSTEM With PeRfect CSI, SNR $=16 \mathrm{~dB}$, AND DIFFERENT DIVERSITY ORDER IN BOTH TIME AND FREQUENCY DOMAINS

\begin{tabular}{|c|c|c|c|}
\hline \multicolumn{4}{|c|}{ DIVERSITY ORDER $=\mathbf{1}:\left(N=1, f_{n d}=10^{-4}\right)$} \\
\hline \multicolumn{4}{|c|}{$\begin{array}{c}\mathrm{psnr}=27.28 \mathrm{~dB} \\
R_{r c p c}=0.36, R_{r s}=0.91 \\
R_{t o t}=0.33\end{array}$} \\
\hline \multicolumn{4}{|c|}{ DIVERSITY ORDER $=2$} \\
\hline \multicolumn{2}{|c|}{$\begin{array}{c}\mathcal{D}_{f}=2, \mathcal{D}_{t}=1 \quad\left(N=2, f_{n d}=10^{-4}\right) \\
\text { psnr }=29.49 \mathrm{~dB} \\
R_{r c p c}=0.73, R_{r s}=0.69 \\
R_{t o t}=0.50\end{array}$} & \multicolumn{2}{|c|}{$\begin{array}{c}\mathcal{D}_{f}=1, \mathcal{D}_{t} \sim 2 \quad\left(N=1, f_{n d}=3,3 \cdot 10^{-3}\right) \\
\text { psnr }=30.71 \mathrm{~dB} \\
R_{r c p c}=0.53, R_{r s}=0.95 \\
R_{\text {tot }}=0.51\end{array}$} \\
\hline \multicolumn{4}{|c|}{ DIVERSITY ORDER $=4$} \\
\hline $\begin{array}{c}\mathcal{D}_{f}=4, \mathcal{D}_{t}=1 \\
\left(N=4, f_{n d}=10^{-4}\right) \\
\mathrm{psnr}=30.55 \mathrm{~dB} \\
R_{r c p c}=0.89, R_{r s}=0.60 \\
R_{\text {tot }}=0.54\end{array}$ & \multicolumn{2}{|c|}{$\begin{array}{c}\mathcal{D}_{f}=2, \mathcal{D}_{t} \sim 2 \\
\left(N=2, f_{n d}=3,3 \cdot 10^{-3}\right) \\
\operatorname{psnr}=30.96 \mathrm{~dB} \\
R_{r c p c}=0.61, R_{r s}=0.89 \\
R_{t o t}=0.54\end{array}$} & $\begin{array}{c}\mathcal{D}_{f}=1, \mathcal{D}_{t} \sim 4 \\
\left(N=1, f_{n d}=8 \cdot 10^{-3}\right) \\
\text { psnr }=31.38 \mathrm{~dB} \\
R_{r c p c}=0.67, R_{r s}=0.92 \\
R_{t o t}=0.61\end{array}$ \\
\hline \multicolumn{4}{|c|}{ DIVERSITY ORDER $=8$} \\
\hline $\begin{array}{c}\mathcal{D}_{f}=8, \mathcal{D}_{t}=1 \\
\left(N=8, f_{n d}=10^{-4}\right) \\
\mathrm{psnr}=31.23 \mathrm{~dB} \\
R_{r c p c}=0.90, R_{r s}=0.65 \\
R_{t o t}=0.58\end{array}$ & $\begin{array}{c}\mathcal{D}_{f}=4, \mathcal{D}_{t} \sim 2 \\
\left(N=4, f_{n d}=3.3 \cdot 10^{-3}\right) \\
\operatorname{psnr}=31.22 \mathrm{~dB} \\
R_{r c p c}=0.73, R_{r s}=0.78 \\
R_{t o t}=0.57\end{array}$ & $\begin{array}{c}\mathcal{D}_{f}=2, \mathcal{D}_{t} \sim 4 \\
\left(N=2, f_{n d}=8 \cdot 10^{-3}\right) \\
\mathrm{psnr}=31.59 \mathrm{~dB} \\
R_{r c p c}=0.73, R_{r s}=0.83 \\
R_{t o t}=0.60\end{array}$ & $\begin{array}{c}\mathcal{D}_{f}=1, \mathcal{D}_{t} \sim 8 \\
\left(N=1, f_{n d}=1.5 \cdot 10^{-2}\right) \\
\text { psnr }=32.01 \mathrm{~dB} \\
R_{r c p c}=0.73, R_{r s}=0.92 \\
R_{t o t}=0.67\end{array}$ \\
\hline \multicolumn{4}{|c|}{ DIVERSITY ORDER $=16$} \\
\hline $\begin{array}{c}\mathcal{D}_{f}=16, \mathcal{D}_{t}=1 \\
\left(N=16, f_{n d}=10^{-4}\right) \\
\mathrm{psnr}=31.75 \mathrm{~dB} \\
R_{r c p c}=0.89, R_{r s}=0.70 \\
R_{\text {tot }}=0.62\end{array}$ & $\begin{array}{c}\mathcal{D}_{f}=8, \mathcal{D}_{t} \sim 2 \\
\left(N=8, f_{n d}=3.3 \cdot 10^{-3}\right) \\
\operatorname{psnr}=31.64 \mathrm{~dB} \\
R_{r c p c}=0.73, R_{r s}=0.82 \\
R_{t o t}=0.60\end{array}$ & $\begin{array}{c}\mathcal{D}_{f}=4, \mathcal{D}_{t} \sim 4 \\
\left(N=4, f_{n d}=8 \cdot 10^{-3}\right) \\
\text { psnr }=31.82 \mathrm{~dB} \\
R_{r c p c}=0.73, R_{r s}=0.88 \\
R_{t o t}=0.64\end{array}$ & $\begin{array}{c}\mathcal{D}_{f}=1, \mathcal{D}_{t} \sim 16 \\
\left(N=1, f_{n d}=3 \cdot 10^{-2}\right) \\
\text { psnr }=32.37 \mathrm{~dB} \\
R_{r c p c}=0.73, R_{r s}=0.95 \\
R_{\text {tot }}=0.69\end{array}$ \\
\hline \multicolumn{4}{|c|}{ DIVERSITY ORDER $=32$} \\
\hline $\begin{array}{c}\mathcal{D}_{f}=32, \mathcal{D}_{t}=1 \\
\left(N=32, f_{n d}=10^{-4}\right) \\
\mathrm{psnr}=32.13 \mathrm{~dB} \\
R_{r c p c}=0.89, R_{r s}=0.75 \\
R_{t o t}=0.67\end{array}$ & $\begin{array}{c}\mathcal{D}_{f}=16, \mathcal{D}_{t} \sim 2 \\
\left(N=16, f_{n d}=3.3 \cdot 10^{-3}\right) \\
\operatorname{psnr}=32.03 \mathrm{~dB} \\
R_{r c p c}=0.80, R_{r s}=0.81 \\
R_{t o t}=0.64\end{array}$ & $\begin{array}{c}\mathcal{D}_{f}=8, \mathcal{D}_{t} \sim 4 \\
\left(N=8, f_{n d}=8 \cdot 10^{-3}\right) \\
\mathrm{psnr}=32.16 \mathrm{~dB} \\
R_{r c p c}=0.80, R_{r s}=0.83 \\
R_{t o t}=0.66\end{array}$ & $\begin{array}{c}\mathcal{D}_{f}=4, \mathcal{D}_{t} \sim 8 \\
\left(N=4, f_{n d}=1.5 \cdot 10^{-2}\right) \\
\operatorname{psnr}=32.23 \mathrm{~dB} \\
R_{r c p c}=0.73, R_{r s}=0.93 \\
R_{t o t}=0.68\end{array}$ \\
\hline
\end{tabular}

ferent simulated results based on five different RCPC code rates. In each of the cases, the corresponding RS boundary is optimally selected based on the chosen RCPC code rate. Fig. 12(c) shows the result for optimal channel coding for the 2-D time-frequency OFDM block. It can be shown that the choice of the optimal 2-D channel coding which includes the proper selections of RCPC codes in the time domain and RS boundary in the frequency domain turns out to be critical. As can be observed, RCPC code rates higher than 0.66 do not provide sufficient protection against the degradation caused by the imperfect channel estimation and the intercarrier interference that the system experiences at high Doppler. Conversely, when an extremely low code rate is chosen, the system might be over protected, leading to too low a source rate.

In Tables II and III, by defining the total/combined channel code rate as $R_{\text {tot }}=R_{\mathrm{rcpc}} \cdot R_{\mathrm{rs}}$ and the total system diversity order available as $\mathcal{D}_{\text {tot }}=\mathcal{D}_{f} \cdot \mathcal{D}_{t}$, we provide further analysis regarding the proposed 2-D channel coding scheme. In particular, in Table II, the optimal PSNR, $R_{\mathrm{rcpc}}, R_{\mathrm{rs}}$, and $R_{\mathrm{tot}}$ are presented for a system with SNR $=16 \mathrm{~dB},{ }^{4}$ perfect CSI and different diversity orders in both the time and frequency domains. In each row, we keep $\mathcal{D}_{\text {tot }}$ fixed and investigate the corresponding performance for different combinations of $\mathcal{D}_{f}$ and $\mathcal{D}_{t}$. To provide a specific comparison, consider systems with $\mathcal{D}_{\text {tot }}$ equal to four. Obviously, this can be achieved with three

\footnotetext{
${ }^{4}$ Although only SNR $=16 \mathrm{~dB}$ is reported, we also considered other SNR values and behavior similar to the one for $\mathrm{SNR}=16 \mathrm{~dB}$ was observed.
}

different combinations: $\left(\mathcal{D}_{f}=4, \mathcal{D}_{t}=1\right),\left(\mathcal{D}_{f}=2, \mathcal{D}_{t}=2\right)$, and $\left(\mathcal{D}_{f}=1, \mathcal{D}_{t}=4\right)$. Generally, as expected, the higher the total diversity, the better is the quality of the received image, and the less is the required redundancy, which is reflected by the increase in combined channel coding rate $R_{\text {tot }}$. Comparing a system with no diversity to a system with $\mathcal{D}=2$, a substantial gain in terms of PSNR can be observed (at least $2 \mathrm{~dB}$ ), although the gain diminishes with increasing diversity orders. This is because, although the error rate of a wireless communication system is generally a strictly decreasing function of the order of diversity, the gain diminishes with increasing order of diversity [37]. What is worth noting from the table is the behavior of optimal PSNR and $R_{\text {tot }}$ for a constant total diversity order. Specifically, it can be seen that for a given $\mathcal{D}_{\text {tot }}$, with perfect CSI, both the optimal PSNR and the $R_{\text {tot }}$ are roughly constant for all the possible combinations of $\mathcal{D}_{f}$ and $\mathcal{D}_{t}$, independent of whether the diversity gain comes from the frequency domain or the time domain.

In Table III, we provide a similar study for a system with imperfect CSI. Similarly to the observation above, we see an enhancement of the performance with an increase in the total diversity. However, for a fixed $\mathcal{D}_{\text {tot }}$, moving from slow fading to fast fading results in a decrease of the optimal PSNR, because of the channel estimation errors. Thus, for a fixed total order of diversity, the system with maximum frequency diversity order performs better than the system with maximum time diversity order. 
TABLE III

Optimal PSNR, $R_{\text {rcpc }}, R_{\mathrm{rs}}$, AND $R_{\text {tot }}$ FOR A SYSTEM With IMPERFECT CSI, SNR $=16 \mathrm{~dB}$ AND DIFFERENT DIVERSITY ORDER IN BOTH TIME AND FREQUENCY DOMAINS

\begin{tabular}{|c|c|c|c|}
\hline \multicolumn{4}{|c|}{ DIVERSITY ORDER $=\mathbf{1}:\left(N=1, f_{n d}=10^{-4}\right)$} \\
\hline \multicolumn{4}{|c|}{$\begin{array}{c}\mathrm{psnr}=26.15 \mathrm{~dB} \\
R_{r c p c}=0.33, R_{r s}=0.90 \\
R_{t o t}=0.30\end{array}$} \\
\hline \multicolumn{4}{|c|}{ DIVERSITY ORDER $=2$} \\
\hline \multicolumn{2}{|c|}{$\begin{array}{c}\mathcal{D}_{f}=2, \mathcal{D}_{t}=1 \quad\left(N=2, f_{n d}=10^{-4}\right) \\
\text { psnr }=28.75 \mathrm{~dB} \\
R_{r c p c}=0.61, R_{r s}=0.67 \\
R_{\text {tot }}=0.41\end{array}$} & \multicolumn{2}{|c|}{$\begin{array}{c}\mathcal{D}_{f}=1, \mathcal{D}_{t} \sim 2 \quad\left(N=1, f_{n d}=3,3 \cdot 10^{-3}\right) \\
\operatorname{psnr}=25.47 \mathrm{~dB} \\
R_{r c p c}=0.33, R_{r s}=0.72 \\
R_{t o t}=0.24\end{array}$} \\
\hline \multicolumn{4}{|c|}{ DIVERSITY ORDER $=4$} \\
\hline $\begin{array}{c}\mathcal{D}_{f}=4, \mathcal{D}_{t}=1 \\
\left(N=4, f_{n d}=10^{-4}\right) \\
\mathrm{psnr}=30.00 \mathrm{~dB} \\
R_{r c p c}=0.80, R_{r s}=0.58 \\
R_{t o t}=0.47\end{array}$ & \multicolumn{2}{|c|}{$\begin{array}{c}\mathcal{D}_{f}=2, \mathcal{D}_{t} \sim 2 \\
\left(N=2, f_{n d}=3,3 \cdot 10^{-3}\right) \\
\mathrm{psnr}=27.06 \mathrm{~dB} \\
R_{r c p c}=0.33, R_{r s}=0.70 \\
R_{t o t}=0.23\end{array}$} & $\begin{array}{c}\mathcal{D}_{f}=1, \mathcal{D}_{t} \sim 4 \\
\left(N=1, f_{n d}=8 \cdot 10^{-3}\right) \\
\mathrm{psnr}=28.13 \mathrm{~dB} \\
R_{r c p c}=0.33, R_{r s}=0.87 \\
R_{\text {tot }}=0.28\end{array}$ \\
\hline \multicolumn{4}{|c|}{ DIVERSITY ORDER $=8$} \\
\hline $\begin{array}{c}\mathcal{D}_{f}=8, \mathcal{D}_{t}=1 \\
\left(N=8, f_{n d}=10^{-4}\right) \\
\mathrm{psnr}=30.74 \mathrm{~dB} \\
R_{r c p c}=0.89, R_{r s}=0.59 \\
R_{t o t}=0.52\end{array}$ & $\begin{array}{c}\mathcal{D}_{f}=4, \mathcal{D}_{t} \sim 2 \\
\left(N=4, f_{n d}=3.3 \cdot 10^{-3}\right) \\
\mathrm{psnr}=27.72 \mathrm{~dB} \\
R_{r c p c}=0.36, R_{r s}=0.66 \\
R_{t o t}=0.24\end{array}$ & $\begin{array}{c}\mathcal{D}_{f}=2, \mathcal{D}_{t} \sim 4 \\
\left(N=2, f_{n d}=8 \cdot 10^{-3}\right) \\
\operatorname{psnr}=28.48 \mathrm{~dB} \\
R_{r c p c}=0.36, R_{r s}=0.82 \\
R_{t o t}=0.29\end{array}$ & $\begin{array}{c}\mathcal{D}_{f}=1, \mathcal{D}_{t} \sim 8 \\
\left(N=1, f_{n d}=1.5 \cdot 10^{-2}\right) \\
\operatorname{psnr}=28.94 \mathrm{~dB} \\
R_{r c p c}=0.36, R_{r s}=0.90 \\
R_{t o t}=0.33\end{array}$ \\
\hline \multicolumn{4}{|c|}{ 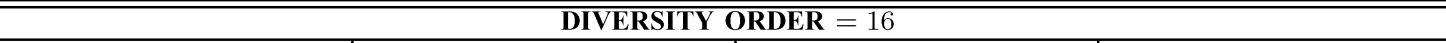 } \\
\hline $\begin{array}{c}\mathcal{D}_{f}=16, \mathcal{D}_{t}=1 \\
\left(N=16, f_{n d}=10^{-4}\right) \\
\mathrm{psnr}=31.33 \mathrm{~dB} \\
R_{r c p c}=0.89, R_{r s}=0.65 \\
R_{t o t}=0.58\end{array}$ & $\begin{array}{c}\mathcal{D}_{f}=8, \mathcal{D}_{t} \sim 2 \\
\left(N=8, f_{n d}=3.3 \cdot 10^{-3}\right) \\
\text { psnr }=28.22 \mathrm{~dB} \\
R_{r c p c}=0.36, R_{r s}=0.71 \\
R_{t o t}=0.26\end{array}$ & $\begin{array}{c}\mathcal{D}_{f}=4, \mathcal{D}_{t} \sim 4 \\
\left(N=4, f_{n d}=8 \cdot 10^{-3}\right) \\
\operatorname{psnr}=28.75 \mathrm{~dB} \\
R_{r c p c}=0.36, R_{r s}=0.83 \\
R_{t o t}=0.30\end{array}$ & $\begin{array}{c}\mathcal{D}_{f}=1, \mathcal{D}_{t} \sim 16 \\
\left(N=1, f_{n d}=3 \cdot 10^{-2}\right) \\
\mathrm{psnr}=29.79 \mathrm{~dB} \\
R_{r c p c}=0.44, R_{r s}=0.88 \\
R_{t o t}=0.39\end{array}$ \\
\hline \multicolumn{4}{|c|}{ DIVERSITY ORDER $=32$} \\
\hline $\begin{array}{c}\mathcal{D}_{f}=32, \mathcal{D}_{t}=1 \\
\left(N=32, f_{n d}=10^{-4}\right) \\
\mathrm{psnr}=31.77 \mathrm{~dB} \\
R_{r c p c}=0.89, R_{r s}=0.70 \\
R_{t o t}=0.62\end{array}$ & $\begin{array}{c}\mathcal{D}_{f}=16, \mathcal{D}_{t} \sim 2 \\
\left(N=16, f_{n d}=3.3 \cdot 10^{-3}\right) \\
\mathrm{psnr}=28.70 \mathrm{~dB} \\
R_{r c p c}=0.44, R_{r s}=0.66 \\
R_{t o t}=0.29\end{array}$ & $\begin{array}{c}\mathcal{D}_{f}=8, \mathcal{D}_{t} \sim 4 \\
\left(N=8, f_{n d}=8 \cdot 10^{-3}\right) \\
\operatorname{psnr}=29.23 \mathrm{~dB} \\
R_{r c p c}=0.44, R_{r s}=0.75 \\
R_{t o t}=0.33\end{array}$ & $\begin{array}{c}\mathcal{D}_{f}=4, \mathcal{D}_{t} \sim 8 \\
\left(N=4, f_{n d}=1.5 \cdot 10^{-2}\right) \\
\text { psnr }=29.26 \mathrm{~dB} \\
R_{r c p c}=0.44, R_{r s}=0.77 \\
R_{t o t}=0.34\end{array}$ \\
\hline
\end{tabular}

\section{A. Rule of Thumb}

The key inferences regarding system design that can be made from this work can be obtained from Tables II and III. Table II corresponds to perfect CSI, and it is seen from that table that for net diversity orders between 2 and 32, the optimal net code rates vary from about 0.5 to 0.7 . Further, the specific breakdown of diversity between frequency diversity and time diversity, for a given net diversity, causes little change in the optimal net code rate. Thus, when one has accurate channel estimates, one can design a robust system simply by knowing the net diversity order and choosing the composite code rate accordingly. When the CSI becomes imperfect (Table III), the net code rates become noticeably lower, varying from about 0.25 to 0.6 . Further, the optimal composite code rate cannot be accurately estimated by the net diversity order. Rather, the specific breakdown of diversity between frequency diversity and time diversity can cause the optimal code rate to vary by a factor of two or more. A design rule of thumb from the data in Table III is that, except for the case of time diversity equal to 1 (when the optimal RCPC code rate is around 0.8 or 0.9 ), in all other cases the RCPC code rate lies between 0.33 and 0.44 , very much lower than the RS code rate (typically about half of it).

\section{CONCLUSION}

We studied channel coding in a 2-D time-frequency resource block of an OFDM system. In particular, we used symmetric $n$-channel FEC-based multiple descriptions based on the diversity order in the frequency domain. In the time domain, a concatenation of RCPC codes and CRC codes was employed to protect individual descriptions. We studied the performance of the proposed system in a doubly-selective channel with channel estimation errors. In a slow-fading environment, it was shown that as the frequency diversity order, increases, the optimal $R_{\text {rcpc }}$ increases and the delivered image quality improves accordingly On the other hand, in a fast-fading environment, the optimal $R_{\text {rcpc }}$ is relatively insensitive to the frequency diversity order, while the performance is limited by the channel estimation errors and ICI. It was also illustrated that the advantages of UEP protection diminishes as the frequency diversity order increases in both slow and fast fading environment. Thus, since both the optimal $R_{\mathrm{rcpc}}$ and $R_{\mathrm{rs}}$ vary depending on the channel conditions, a system can be robust only employing a 2-D channel coding adaptable to both time and frequency diversity orders. Lastly, we illustrated that the bursty nature of a slow fading environment can lead to a higher application layer throughput and thereby deliver a better image quality, while the scattered error pattern in a fast fading environment may lead to poor image quality.

\section{APPENDIX}

For a Rayleigh fading process $r(t)$, consider a simple twostate threshold model [34] with $R_{t h}$ being the threshold for the Rayleigh fading signal. If the signal level is above $R_{t h}$ (strong 
fade), the channel is considered to be in the good state, in which case the probability of receiving the information is equal to 1 , while if the signal level is below $R_{t h}$ (deep fade), the probably of receiving the particular information bit is equal to 0 . Let us further assume that $\tau_{f}, \tau_{n f}$ and $\tau_{s}$ are the deep fade duration, the strong fade duration and the deep fade interarrival intervals, respectively, so that

$$
\tau_{s}=\tau_{f}+\tau_{n f}
$$

The average deep fade interarrival interval is the inverse of the level crossing rate (the expected rate at which the signal crosses the $\left.R_{t h}\right)$, defined as [36]

$$
\overline{\tau_{s}}=\frac{1}{\sqrt{2 \pi} f_{D} \rho e^{-\rho^{2}}}
$$

where $f_{D}$ is the maximum Doppler frequency, $\rho^{2}=$ $\left(R_{t h} / R_{r m s}\right)^{2}$ is the inverse of the fade margin and $R_{r m s}$ is the root mean square of the fading signal. Denoting by $N_{r}$ the level crossing rate, the average deep fade duration is defined as [36]

$$
\overline{\tau_{f}}=\frac{P\left(r(t) \leq R_{t h}\right)}{N_{r}}=\frac{e^{\rho^{2}}-1}{\sqrt{2 \pi} f_{D} \rho}
$$

so that the average strong fade duration can be expressed as

$$
\overline{\tau_{n f}}=\overline{\tau_{s}}-\overline{\tau_{f}}=\frac{1}{\sqrt{2 \pi} \rho f_{D}} .
$$

Note that the ratio between the average strong fade duration and the average deep fade duration is not a function of the Doppler spread. Thus, increasing $f_{D}$ causes a reduction of the average deep fade interarrival interval $\left(\overline{\tau_{s}}\right)$, but also a shorter deep fade duration, as can be observed from (10) and (11), respectively. Hence, faster fading produces deep fade events that are shorter in duration but occur more frequently.

Since $r(t)$ is a Rayleigh random process, $r^{2}(t)$ is a $\chi^{2}$ process and thus its asymptotic ${ }^{5}$ level down-crossing rate forms a Poisson process [38]. From the properties of Poisson random variables [39], it follows that

$$
P_{\tau_{s}}(\tau)=\operatorname{Prob}\left(\tau_{s} \leq \tau\right)=1-\exp \left(-\frac{\tau}{\overline{\tau_{s}}}\right)
$$

and we can define the probability of having $k$ deep fade arrivals within an interval of $T_{P L}$ seconds as

$$
\operatorname{Prob}\left\{\mathcal{K}\left(T_{P L}\right)=k\right\}=\frac{\left(T_{P L} / \overline{\tau_{s}}\right)^{k} e^{-T_{P L} / \overline{\tau_{s}}}}{k !}
$$

where $T_{P L}$ corresponds to the duration of a packet, and $\mathcal{K}\left(T_{P L}\right)$ is a random variable representing the number of deep fade arrivals in $T_{P L}$ seconds.

\footnotetext{
${ }^{5}$ We use "asymptotic level down-crossing" to mean the level crossing of a very low threshold $\left(R_{t h} \rightarrow 0\right)$ [38].
}

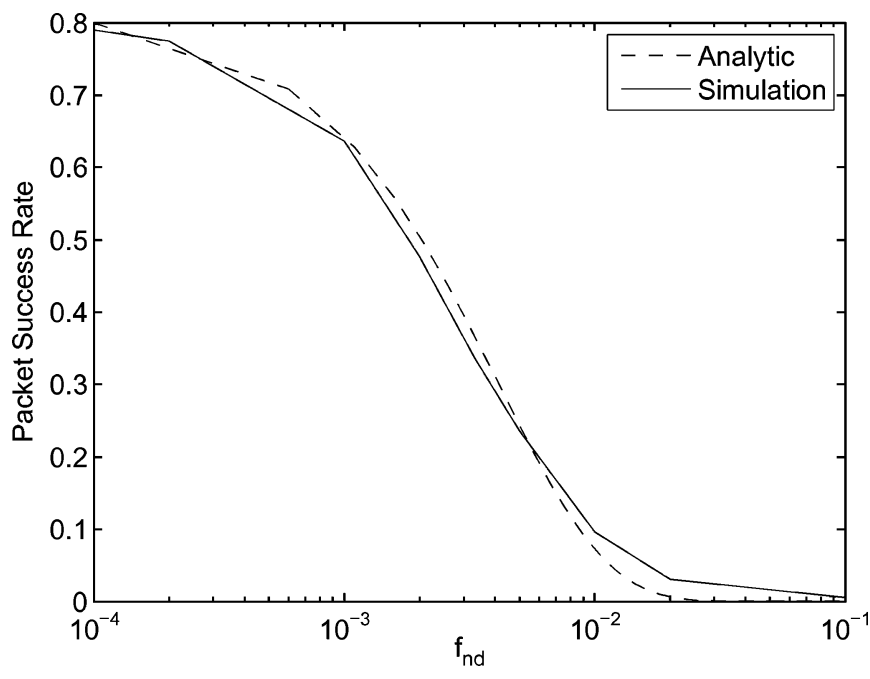

Fig. 13. Simulation and analytic results for the packet success rate for an uncoded system with different $f_{n d}$ with $\operatorname{SNR}\left(R_{t h}\right)=9 \mathrm{~dB}$.

Thus, in an uncoded system, the probability of having a packet correctly received $\left(P_{\text {succ }}\right)$ is

$$
\begin{aligned}
P_{\text {succ }} & =P_{\text {good }} \cdot \operatorname{Prob}\left(\mathcal{K}\left(T_{P L}\right)=0\right) \\
& =\exp \left(-\rho^{2}-\frac{T_{P L}}{\overline{\tau_{s}}}\right)
\end{aligned}
$$

where

$$
\begin{aligned}
P_{\text {good }} & =\operatorname{Prob}(\text { packet starts in good fade }) \\
& =P\left(r(t)>R_{t h}\right)=\exp \left(-\rho^{2}\right)
\end{aligned}
$$

is the probability that a packet starts in the good state. From (15), it can be seen that the packet success rate probability decreases with decreasing interarrival time of the deep fades due to the fact that deep fading intervals are shorter but occur more frequently. In Fig. 13, we show both the simulation results and analytical results with different fade rates. As can be seen from the figure, the simulation and analytical results closely track one another. In particular, the packet success rate decreases with increasing fade rate. This explains the performance of Fig. 8, in which the PSNR of systems with either perfect CSI or imperfect CSI decreases monotonically with increasing fade rates.

\section{REFERENCES}

[1] P. G. Sherwood and K. Zeger, "Progressive image coding for noisy channels," IEEE Signal Process. Lett., vol. 4, no. 7, pp. 191-198, Jul. 1997.

[2] P. Cosman, J. Rogers, P. G. Sherwood, and K. Zeger, "Combined forward error control and packetized zerotree wavelet encoding for transmission of images over varying channels," IEEE Trans. Image Process., vol. 9, no. 6, pp. 132-140, Jun. 2000.

[3] P. G. Sherwood and K. Zeger, "Error protection for progressive image transmission over memoryless and fading channels," IEEE Trans. Commun., vol. 46, no. 12, Dec. 1998.

[4] A. Albanese, J. Blomer, J. Edmonds, M. Luby, and M. Sudan, "Priority encoded transmission," IEEE Trans. Inf. Theory, vol. 46, no. 6, pp. 1737-1744, Nov. 1996. 
[5] A. E. Mohr, E. A. Riskin, and R. E. Ladner, "Graceful degradation over packet erasure channels through forward error correction," in Proc. IEEE Data Compression Conf., Snowbird, UT, Mar. 1999, pp. 92-101.

[6] R. Puri, K.-W. Lee, K. Ramchandran, and V. Bharghavan, “An integrated source transcoding and congestion control paradigm for video streaming in the Internet," IEEE Trans. Multimedia, vol. 3, no. 1, pp. 18-32, Mar. 2001.

[7] D. G. Sachs, R. Anand, and K. Ramchandran, "Wireless image transmission using multiple-description based concatenated codes," in Proc. SPIE, San Jose, CA, Jan. 2000, vol. 3974, pp. 300-311.

[8] J. N. Laneman, E. Martinian, G. W. Wornell, J. G. Apostolopoulos, and S. J. Wee, "Comparing application- and physical-layer approaches to diversity on wireless channels," in Proc. ICC, Anchorage, AK, May 2003, vol. 4, pp. 2678-2682.

[9] Y. S. Chan, P. C. Cosman, and L. B. Milstein, "A cross-layer diversity technique for multi-carrier OFDM multimedia networks," IEEE Trans. Image Process., vol. 15, no. 4, pp. 833-847, Apr. 2006.

[10] Y. Li, L. J. Cimini, Jr., and N. R. Sollenberger, "Robust channel estimation for ofdm systems with rapid dispersive fading channels," IEEE Trans. Commun., vol. 46, no. 7, pp. 902-915, Jul. 1998.

[11] Y. Li, "Pilot-symbol-aided channel estimation for OFDM in wireless systems," IEEE Trans. Veh. Technol., vol. 49, no. 4, pp. 1207-1215, Jun. 2000.

[12] X. Cai and G. B. Giannakis, "Adaptive PSAM accounting for channel estimation and prediction errors," IEEE Trans. Wireless Commun., vol. 4, no. 1, pp. 246-256, Jan. 2005.

[13] A. Stefanov and T. M. Duman, "Turbo-coded modulation for systems with transmit and receive antenna diversity over block fading channels: System model, decoding approaches and practical considerations," IEEE J. Sel. Areas Commun., vol. 19, no. 5, pp. 958-968, May 2001.

[14] R. M. Buehrer and N. A. Kumar, "The impact of channel estimation error on space-time block codes," in Proc. VTC, Vancouver, BC, Canada, Sep. 2002, vol. 3, pp. 1921-1925.

[15] V. Tarokh, N. Seshadri A. Naguib, and A. R. Calderbank, "Space-time codes for high data rate wireless communication: Performance criteria in the presence of channel estimation errors, mobility, and multiple paths," IEEE Trans. Commun., vol. 47, no. 2, pp. 199-207, 1999.

[16] J. Song and K. J. R. Liu, "Robust progressive image transmission over OFDM systems using space-time block code," IEEE Trans. Multimedia, vol. 4, no. 3, pp. 394-406, Sep. 2002.

[17] Y. Sun, Z. Xiong, and X. Wang, "Scalable image transmission over differentially space-time coded OFDM systems," in Proc. GlobeCom, Taipei, Taiwan, R.O.C., Nov. 2002, vol. 1, pp. 379-383.

[18] F. Masoud, S. Cho, and W. A. Pearlman, "Robust image transmission using a new joint source channel coding algorithm and dual adaptive OFDM," in Proc. GlobeCom, San Jose, CA, Jan. 2004, vol. 5308, pp. 636-646.

[19] R. J. McEliece and W. E. Stark, "Channels with block interference," IEEE Trans. Inf. Theory, vol. IT-30, no. 1, pp. 44-53, Jan. 1984.

[20] Y. R. Zheng and C. Xiao, "Simulation models with correct statistical properties for Rayleigh fading channels," IEEE Trans. Commun., vol. 51, no. 6, pp. 920-928, Jun. 2003.

[21] T. S. Rappaport, Wireless Communications: Principles \& Practice. Upper Saddle River, NJ: Prentice-Hall, 1996.

[22] M. Russell and G. L. Stuber, "Interchannel interference analysis of OFDM in a mobile environment," in Proc. VTC, Chicago, IL, Jul. 1995, vol. 2, pp. 820-824.

[23] P. Robertson and S. Kaiser, "The effects of doppler spreads in OFDM(A) mobile radio systems," in Proc. VTC, Amsterdam, The Netherlands, Fall, 1999, vol. 1, pp. 329-333.

[24] T. Wang, J. G. Proakis, E. Masry, and J. R. Zeidler, "Performance degradation of OFDM systems due to doppler spreading," IEEE Trans. Wireless Commun., vol. 5, no. 6, pp. 1422-1432, Jun. 2006.

[25] J. K. Cavers, "An analysis of pilot symbol assisted modulation for rayleigh fading channels," IEEE Trans. Veh. Technol., vol. 40, no. 4, pp. 686-693, Nov. 1991.

[26] X. Tang, M.-S. Alouini, and A. J. Goldsmith, "Effect of channel estimation error on M-QAM BER performance in Rayleigh fading," IEEE Trans. Commun., vol. 47, no. 12, pp. 1856-1864, Dec. 1999.

[27] R. Negi and J. Cioffi, "Pilot tone selection for channel estimation in a mobile OFDM system," IEEE Trans. Consum. Electron., vol. 44, no. 3, pp. 1122-1128, Aug. 1998.
[28] M.-A. R. Baissas and A. M. Sayeed, "Pilot-based estimation of time-varying multipath channels for coherent CDMA receivers," IEEE Trans. Signal Process., vol. 50, no. 8, pp. 2037-2049, Aug. 2002.

[29] M. Dong, L. Tong, and B. M. Sadler, "Optimal insertion of pilot symbols for transmissions over time-varying flat fading channels," IEEE Trans. Signal Process., vol. 52, no. 5, pp. 1403-1418, May 2004.

[30] S. M. Kay, Fundamentals of Statistical Processing, Volume I: Estimation Theory. Upper Saddle River, NJ: Prentice-Hall, 1993.

[31] A. Said and W. A. Pearlman, "A new, fast, and efficient image codec based on set partitioning in hierarchical trees," IEEE Trans. Circuits Syst. Video Technol., vol. 6, pp. 243-249, Jun. 1996.

[32] S.-S. Tan, M. Rim, P. C. Cosman, and L. B. Milstein, "Adaptive modulation for OFDM-based multiple description progressive image transmission," in Proc. IEEE GlobeCom, New Orleans, LA, Nov./Dec. 2008.

[33] P. Frenger, P. Orten, T. Ottosson, and A. Svensson, Multi-Rate Convolutional Codes, Technical Report No. 21 Dept. Information Theory, Chalmers Univ. Technology, Apr. 1998, Tech. Rep..

[34] M. Zorzi, R. R. Rao, and L. B. Milstein, "Error statistics in data transmission over fading channels," IEEE Trans. Commun., vol. 46, no. 11, pp. 1468-1477, Nov. 1998.

[35] J. Lai and N. B. Mandayam, "Performance of reed-solomon codes for hybrid-arq over rayleigh fading channels under imperfect interleaving," IEEE Trans. Commun., vol. 48, no. 10, pp. 1650-1659, Oct. 2000.

[36] W. C. Jakes, Microwave Mobile Communications. New York: Wiley, 1994.

[37] W. Xu and L. B. Milstein, "On the performance of multicarrier RAKE systems," IEEE Trans. Commun., vol. 49, no. 10, pp. 1812-1823, Oct. 2001.

[38] M. Aronowich and R. J. Adler, "Extrema and level crossings of $\chi^{2}$ processes," Adv. Appl. Probab., vol. 18, no. 4, pp. 901-920, Dec. 1986.

[39] A. Papoulis, Probability, Random Variables and Stochastic Processes, 3rd ed. New York: McGraw-Hill, 1991.

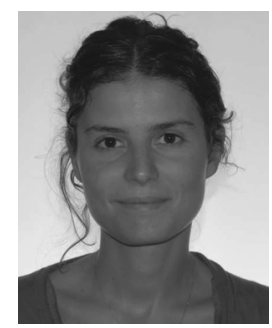

Laura Toni (S'06) received the B.S. and M.S. degrees (with honors) in electrical engineering from the University of Bologna, Italy, in 2003 and 2005, respectively, where she is currently pursuing the Ph.D. degree.

In 2005, she joined the Department of Electronics, Informatics and Systems, University of Bologna, to develop her research activity in the area of wireless communications. During 2007, she was a Visiting Scholar at the University of California at San Diego, La Jolla. In June 2009, she joined the TERA department at the Italian Institute of Technology (IIT), Genova, Italy. Her research interests are in the areas of image and video processing, and wireless communications.

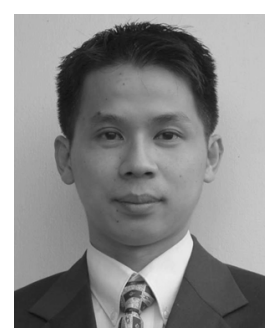

Yee Sin Chan ( $\left.\mathrm{S}^{\prime} 00-\mathrm{M}^{\prime} 03\right)$ received the B.Eng. (Hons.) degree in electrical and electronic engineering from the University of Hong Kong (HKU), the M.Phil. degree in physics from the Hong Kong University of Science and Technology (HKUST), and the M.Sc. and Ph.D. degrees in electrical engineering from Rensselaer Polytechnic Institute, Troy, NY

From 2003 to 2005, he was a Postdoctoral Research Scientist at the University of California at San Diego, La Jolla, CA. From 2005 to 2006, he was a Visiting Assistant Professor in the Department of Electrical and Computer Engineering, University of Miami, Coral Gables, FL. He was an Electrical Engineer at NextWave Broadband, San Diego, CA, in 2006. Since December 2006, he has been with Verizon. Currently, he is a Distinguished Member of Technical Staff at Verizon Corporate Technology, Walnut Creek, CA. His research interests lie in the areas of communications, signal processing, and information theory.

Dr. Chan has received several awards, including the Sir Edward Youde Fellowship from the Hong Kong Government, the Y. L. Liu Fellowship from RPI, and the Joyce M. Kuok Scholarship from HKU. He is a member of Sigma Xi. 


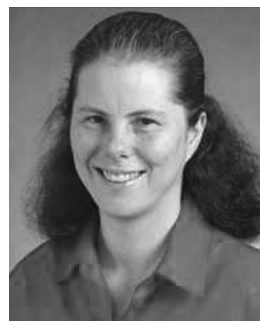

Pamela C. Cosman (S'88-M'93-SM'00-F'08) received the B.S. degree with Honors in electrical engineering from the California Institute of Technology, Pasadena, in 1987, and the M.S. and Ph.D. degrees in electrical engineering from Stanford University, Stanford, CA, in 1989 and 1993, respectively.

She was an NSF postdoctoral fellow at Stanford University and a Visiting Professor at the University of Minnesota during 1993-1995. In 1995, she joined the faculty of the Department of Electrical and Computer Engineering, University of California at San Diego, La Jolla, where she is currently a Professor. She was the Director of the Center for Wireless Communications from 2006 to 2008. Her research interests are in the areas of image and video compression and processing and wireless communications.

Dr. Cosman is the recipient of the ECE Departmental Graduate Teaching Award (1996), a Career Award from the National Science Foundation (1996-1999), a Powell Faculty Fellowship (1997-1998), and a Globecom 2008 Best Paper Award. She was a guest editor of the June 2000 special issue of the IEEE JOURNAL ON SELECTED AREAS IN COMMUNICATIONS on "Error-resilient image and video coding," and was the Technical Program Chair of the 1998 Information Theory Workshop in San Diego. She was an associate editor of the IEEE COMMUNICATIONS LETTERS (1998-2001), and an associate editor of the IEEE Signal PROCESSING LetTers (2001-2005). She was a senior editor (2003-2005) and is now the Editor-in-Chief of the IEEE JOURNAL ON Selected AREas in Communications. She is a member of Tau Beta Pi and Sigma Xi.

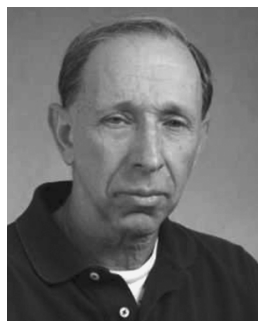

Laurence B. Milstein (S'66-M'68-SM'77-F'85) received the B.E.E. degree from the City College of New York, New York, in 1964, and the M.S. and $\mathrm{Ph} . \mathrm{D}$. degrees in electrical engineering from the Polytechnic Institute of Brooklyn, Brooklyn, NY, in 1966 and 1968, respectively.

From 1968 to 1974 , he was with the Space and Communications Group of Hughes Aircraft Company, and from 1974 to 1976, he was a member of the Department of Electrical and Systems Engineering, Rensselaer Polytechnic Institute, Troy, NY. Since 1976, he has been with the Department of Electrical and Computer Engineering, University of California at San Diego, La Jolla, where he is the Ericsson Professor of Wireless Communications Access Techniques and former Department Chairman, working in the area of digital communication theory with special emphasis on spread-spectrum communication systems. $\mathrm{He}$ has also been a consultant to both government and industry in the areas of radar and communications.

Dr. Milstein was an Associate Editor for Communication Theory for the IEEE TRANSACTIONS ON COMMUNICATIONS, an Associate Editor for Book Reviews for the IEEE TRANSACTIONS ON INFORMATION THEORY, an Associate Technical Editor for the IEEE Communications Magazine, and the Editor-in-Chief of the IEEE Journal on Selected AREAS In Communications. He was the Vice President for Technical Affairs in 1990 and 1991 of the IEEE Communications Society, and is a former Chair of the IEEE Fellows Selection Committee. $\mathrm{He}$ is a recipient of the 1998 Military Communications Conference Long Term Technical Achievement Award, an Academic Senate 1999 UCSD Distinguished Teaching Award, an IEEE Third Millennium Medal in 2000, the 2000 IEEE Communication Society Armstrong Technical Achievement Award, and various prize paper awards, including the 2002 MILCOM Fred Ellersick Award. 